\title{
Extremal Graph Theory for Metric Dimension and Diameter*
}

\author{
Carmen Hernando ${ }^{\dagger}$ \\ Departament de Matemàtica Aplicada I \\ Universitat Politècnica de Catalunya \\ Barcelona, Spain \\ carmen.hernando@upc.edu \\ Ignacio M. Pelayo \\ Departament de Matemàtica Aplicada III \\ Universitat Politècnica de Catalunya \\ Barcelona, Spain \\ ignacio.m.pelayo@upc.edu
}

\author{
Mercè Mora ${ }^{\dagger}$ \\ Departament de Matemàtica Aplicada II \\ Universitat Politècnica de Catalunya \\ Barcelona, Spain \\ merce.mora@upc.edu \\ Carlos Seara $^{\dagger}$ \\ Departament de Matemàtica Aplicada II \\ Universitat Politècnica de Catalunya \\ Barcelona, Spain \\ carlos.seara@upc.edu
}

\author{
David R. Wood ${ }^{\S}$ \\ Department of Mathematics and Statistics \\ The University of Melbourne \\ Melbourne, Australia \\ woodd@unimelb.edu.au
}

Submitted: Jul 31, 2008; Accepted: Feb 11, 2010; Published: Feb 22, 2010

Subject Classification: 05C12 (distance in graphs), 05C35 (extremal graph theory)

Keywords: graph, distance, resolving set, metric dimension, metric basis, diameter, order

\footnotetext{
${ }^{*}$ An extended abstract of this paper was presented at the European Conference on Combinatorics, Graph Theory and Applications (EuroComb '07), Electronic Notes in Discrete Mathematics 29:339-343, 2007.

${ }^{\dagger}$ Research supported by project MTM2009-07242 and Gen. Cat. DGR 2009SGR1040.

${ }^{\ddagger}$ Research supported by projects MTM2008-06620-C03-01 and 2009SGR-1387.

${ }^{\S}$ Supported by a QEII Research Fellowship. Research conducted at the Universitat Politècnica de Catalunya, where supported by a Marie Curie Fellowship under contract MEIF-CT-2006-023865, and by projects MEC MTM2006-01267 and DURSI 2005SGR00692.
} 


\begin{abstract}
A set of vertices $S$ resolves a connected graph $G$ if every vertex is uniquely determined by its vector of distances to the vertices in $S$. The metric dimension of $G$ is the minimum cardinality of a resolving set of $G$. Let $\mathcal{G}_{\beta, D}$ be the set of graphs with metric dimension $\beta$ and diameter $D$. It is well-known that the minimum order of a graph in $\mathcal{G}_{\beta, D}$ is exactly $\beta+D$. The first contribution of this paper is to characterise the graphs in $\mathcal{G}_{\beta, D}$ with order $\beta+D$ for all values of $\beta$ and $D$. Such a characterisation was previously only known for $D \leqslant 2$ or $\beta \leqslant 1$. The second contribution is to determine the maximum order of a graph in $\mathcal{G}_{\beta, D}$ for all values of $D$ and $\beta$. Only a weak upper bound was previously known.
\end{abstract}

\title{
1 Introduction
}

Resolving sets in graphs, first introduced by Slater [36] and Harary and Melter [16], have since been widely investigated [2, 3, 4, 5, 6, 7, 9, 17, 18, 19, 21, 23, 24, 25, 26, 27, 28, 29, 30, 31, 32, 33, 34, 35, 37, 39, 40, 41, 42. They arise in diverse areas including coin weighing problems [11, 15, 20, 22, 38], network discovery and verification [1], robot navigation [21, 35], connected joins in graphs [34], the Djoković-Winkler relation [3], and strategies for the Mastermind game [8, 12, 13, 14, 20].

Let $G$ be a connected graph ${ }^{1}$. A vertex $x \in V(G)$ resolves ${ }^{2}$ a pair of vertices $v, w \in$ $V(G)$ if $\operatorname{dist}(v, x) \neq \operatorname{dist}(w, x)$. A set of vertices $S \subseteq V(G)$ resolves $G$, and $S$ is a resolving set of $G$, if every pair of distinct vertices of $G$ is resolved by some vertex in $S$. Informally, $S$ resolves $G$ if every vertex of $G$ is uniquely determined by its vector of distances to the vertices in $S$. A resolving set $S$ of $G$ with the minimum cardinality is a metric basis of $G$, and $|S|$ is the metric dimension of $G$, denoted by $\beta(G)$.

For positive integers $\beta$ and $D$, let $\mathcal{G}_{\beta, D}$ be the class of connected graphs with metric dimension $\beta$ and diameter $D$. Consider the following two extremal questions:

- What is the minimum order of a graph in $\mathcal{G}_{\beta, D}$ ?

- What is the maximum order of a graph in $\mathcal{G}_{\beta, D}$ ?

\footnotetext{
${ }^{1}$ Graphs in this paper are finite, undirected, and simple. The vertex set and edge set of a graph $G$ are denoted by $V(G)$ and $E(G)$. For vertices $v, w \in V(G)$, we write $v \sim w$ if $v w \in E(G)$, and $v \nsim w$ if $v w \notin E(G)$. For $S \subseteq V(G)$, let $G[S]$ be the subgraph of $G$ induced by $S$. That is, $V(G[S])=S$ and $E(G[S])=\{v w \in E(G): v \in S, w \in S\})$. For $S \subseteq V(G)$, let $G \backslash S$ be the graph $G[V(G) \backslash S]$. For $v \in V(G)$, let $G \backslash v$ be the graph $G \backslash\{v\}$. Now suppose that $G$ is connected. The distance between vertices $v, w \in V(G)$, denoted by $\operatorname{dist}_{G}(v, w)$, is the length (that is, the number of edges) in a shortest path between $v$ and $w$ in $G$. The eccentricity of a vertex $v$ in $G$ is $\operatorname{ecc}_{G}(v):=\max \left\{\operatorname{dist}_{G}(v, w): w \in V(G)\right\}$. We drop the subscript $G$ from these notations if the graph $G$ is clear from the context. The diameter of $G$ is $\operatorname{diam}(G):=\max \{\operatorname{dist}(v, w): v, w \in V(G)\}=\max \{\operatorname{ecc}(v): v \in V(G)\}$. For integers $a \leqslant b$, let $[a, b]:=\{a, a+1, \ldots, b\}$. Undefined terminology can be found in [10].

${ }^{2}$ It will be convenient to also use the following definitions for a connected graph $G$. A vertex $x \in V(G)$ resolves a set of vertices $T \subseteq V(G)$ if $x$ resolves every pair of distinct vertices in $T$. A set of vertices $S \subseteq V(G)$ resolves a set of vertices $T \subseteq V(G)$ if for every pair of distinct vertices $v, w \in T$, there exists a vertex $x \in S$ that resolves $v, w$.
} 
The first question was independently answered by Yushmanov [42], Khuller et al. [21], and Chartrand et al. [5], who proved that the minimum order of a graph in $\mathcal{G}_{\beta, D}$ is $\beta+D$ (see Lemma 2.2. Thus it is natural to consider the following problem:

- Characterise the graphs in $\mathcal{G}_{\beta, D}$ with order $\beta+D$.

Such a characterisation is simple for $\beta=1$. In particular, Khuller et al. [21] and Chartrand et al. [5] independently proved that paths $P_{n}$ (with $n \geqslant 2$ vertices) are the only graphs with metric dimension 1 . Thus $\mathcal{G}_{1, D}=\left\{P_{D+1}\right\}$.

The characterisation is again simple at the other extreme with $D=1$. In particular, Chartrand et al. [5] proved that the complete graph $K_{n}$ (with $n \geqslant 1$ vertices) is the only graph with metric dimension $n-1$ (see Proposition 2.12). Thus $\mathcal{G}_{\beta, 1}=\left\{K_{\beta+1}\right\}$.

Chartrand et al. [5] studied the case $D=2$, and obtained a non-trivial characterisation of graphs in $\mathcal{G}_{\beta, 2}$ with order $\beta+2$ (see Proposition 2.13).

The first contribution of this paper is to characterise the graphs in $\mathcal{G}_{\beta, D}$ with order $\beta+D$ for all values of $\beta \geqslant 1$ and $D \geqslant 3$, thus completing the characterisation for all values of $D$. This result is stated and proved in Section 2 ,

We then study the second question above: What is the maximum order of a graph in $\mathcal{G}_{\beta, D}$ ? Previously, only a weak upper bound was known. In particular, Khuller et al. 21] and Chartrand et al. [5] independently proved that every graph in $\mathcal{G}_{\beta, D}$ has at most $D^{\beta}+\beta$ vertices. This bound is tight only for $D \leqslant 3$ or $\beta=1$.

Our second contribution is to determine the (exact) maximum order of a graph in $\mathcal{G}_{\beta, D}$ for all values of $D$ and $\beta$. This result is stated and proved in Section 3 .

\section{Graphs with Minimum Order}

In this section we characterise the graphs in $\mathcal{G}_{\beta, D}$ with minimum order. We start with an elementary lemma.

Lemma 2.1. Let $S$ be a set of vertices in a connected graph $G$. Then $V(G) \backslash S$ resolves $G$ if and only if every pair of vertices in $S$ is resolved by some vertex not in $S$.

Proof. If $v \in V(G) \backslash S$ and $w$ is any other vertex, then $v$ resolves $v$ and $w$. By assumption every pair of vertices in $S$ is resolved by some vertex in $V(G) \backslash S$.

Lemma 2.1 enables the minimum order of a graph in $\mathcal{G}_{\beta, D}$ to be easily determined.

Lemma 2.2 ([5, 21, 42]). The minimum order of a graph in $\mathcal{G}_{\beta, D}$ is $\beta+D$.

Proof. First we prove that every graph $G \in \mathcal{G}_{\beta, D}$ has order at least $\beta+D$. Let $v_{0}, v_{D}$ be vertices such that $\operatorname{dist}\left(v_{0}, v_{D}\right)=D$. Let $P=\left(v_{0}, v_{1}, \ldots, v_{D}\right)$ be a path of length $D$ in $G$. Then $v_{0}$ resolves $v_{i}, v_{j}$ for all distinct $i, j \in[1, D]$. Thus $V(G) \backslash\left\{v_{1}, \ldots, v_{D}\right\}$ resolves $G$ by Lemma 2.1. Hence $\beta \leqslant|V(G)|-D$ and $|V(G)| \geqslant \beta+D$.

It remains to construct a graph $G \in \mathcal{G}_{\beta, D}$ with order $\beta+D$. Let $G$ be the 'broom' tree obtained by adding $\beta$ leaves adjacent to one endpoint of the path on $D$ vertices. Observe 
that $|V(G)|=\beta+D$ and $G$ has diameter $D$. It follows from Slater's formula [36] for the metric dimension of a tre $\varepsilon^{3}$ that the $\beta$ leaves adjacent to one endpoint of the path are a metric basis of $G$. Hence $G \in \mathcal{G}_{\beta, D}$.

\subsection{Twin Vertices}

The following definitions and lemmas about twin vertices are well known. Let $u$ be a vertex of a graph $G$. The open neighbourhood of $u$ is $N(u):=\{v \in V(G): u v \in E(G)\}$, and the closed neighbourhood of $u$ is $N[u]:=N(u) \cup\{u\}$. Two distinct vertices $u, v$ are adjacent twins if $N[u]=N[v]$, and non-adjacent twins if $N(u)=N(v)$. Observe that if $u, v$ are adjacent twins then $u v \in E(G)$, and if $u, v$ are non-adjacent twins then $u v \notin E(G)$; thus the names are justified 4 . If $u, v$ are adjacent or non-adjacent twins, then $u, v$ are twins. The next lemma follows from the definitions.

Lemma 2.3. If $u, v$ are twins in a connected graph $G$, then $\operatorname{dist}(u, x)=\operatorname{dist}(v, x)$ for every vertex $x \in V(G) \backslash\{u, v\}$.

Corollary 2.4. Suppose that $u, v$ are twins in a connected graph $G$ and $S$ resolves $G$. Then $u$ or $v$ is in $S$. Moreover, if $u \in S$ and $v \notin S$, then $(S \backslash\{u\}) \cup\{v\}$ also resolves $G$.

Lemma 2.5. In a set $S$ of three vertices in a graph, it is not possible that two vertices in $S$ are adjacent twins, and two vertices in $S$ are non-adjacent twins.

Proof. Suppose on the contrary that $u, v$ are adjacent twins and $v, w$ are non-adjacent twins. Since $u, v$ are twins and $v \not w$, we have $u \neq w$. Similarly, since $v, w$ are twins and $u \sim v$, we have $u \sim w$. This is the desired contradiction.

Lemma 2.6. Let $u, v, w$ be distinct vertices in a graph. If $u, v$ are twins and $v, w$ are twins, then $u, w$ are also twins.

Proof. Suppose that $u, v$ are adjacent twins. That is, $N[u]=N[v]$. By Lemma 2.5, $v, w$ are adjacent twins. That is, $N[v]=N[w]$. Hence $N[u]=N[w]$. That is, $u, w$ are adjacent twins. By a similar argument, if $u, v$ are non-adjacent twins, then $v, w$ are non-adjacent twins and $u, w$ are non-adjacent twins.

For a graph $G$, a set $T \subseteq V(G)$ is a twin-set of $G$ if $v, w$ are twins in $G$ for every pair of distinct vertices $v, w \in T$.

Lemma 2.7. If $T$ is a twin-set of a graph $G$, then either every pair of vertices in $T$ are adjacent twins, or every pair of vertices in $T$ are non-adjacent twins.

\footnotetext{
${ }^{3}$ Also see [5, 16, 21] for proofs of Slater's formula.

${ }^{4}$ In the literature, adjacent twins are called true twins, and non-adjacent twins are called false twins. We prefer the more descriptive names, adjacent and non-adjacent.
} 
Proof. Suppose on the contrary that some pair of vertices $v, w \in T$ are adjacent twins, and some pair of vertices $x, y \in T$ are non-adjacent twins. Thus $v, w, x, y$ are distinct vertices by Lemma 2.5. If $v, x$ are adjacent twins then $\{v, x, y\}$ contradict Lemma 2.5. Otherwise $v, x$ are non-adjacent twins, in which case $\{v, w, x\}$ contradict Lemma 2.5.

Twin sets are important in the study of metric dimension because of the following lemma.

Lemma 2.8. Let $T$ be a twin-set of a connected graph $G$ with $|T| \geqslant 3$. Then $\beta(G)=$ $\beta(G \backslash u)+1$ for every vertex $u \in T$.

Proof. Let $u, v, w$ be distinct vertices in T. By Corollary 2.4, there is a metric basis $W$ of $G$ such that $u, v \in W$. Since $u$ has a twin in $G \backslash u$, for all $x, y \in V(G \backslash u)$ we have $\operatorname{dist}_{G}(x, y)=\operatorname{dist}_{G \backslash u}(x, y)$. In particular, $G \backslash u$ is connected. First we prove that $W \backslash\{u\}$ resolves $G \backslash u$. For all distinct vertices $x, y \in V(G \backslash u)$, there is a vertex $s \in W$ such that $\operatorname{dist}_{G}(x, s) \neq \operatorname{dist}_{G}(y, s)$. If $s \neq u$, then $s \in W \backslash\{u\}$ resolves the pair $x, y$. Otherwise, $v$ is a twin of $s=u$ and $\operatorname{dist}_{G \backslash u}(x, v)=\operatorname{dist}_{G}(x, v)=\operatorname{dist}_{G}(x, s) \neq \operatorname{dist}_{G}(y, s)=\operatorname{dist}_{G}(y, v)=$ $\operatorname{dist}_{G \backslash u}(y, v)$. Consequently, $v \in W \backslash\{u\}$ resolves the pair $x, y$. Now suppose that $W^{\prime}$ is a resolving set of $G \backslash u$ such that $\left|W^{\prime}\right|<|W|-1$. For all $x, y \in V(G \backslash u)$, there exists a vertex $s \in W^{\prime}$ such that $\operatorname{dist}_{G \backslash u}(x, s) \neq \operatorname{dist}_{G \backslash u}(y, s)$. Then $W^{\prime} \cup\{u\}$ is a resolving set in $G$ of cardinality less than $|W|$, which contradicts the fact that $W$ is a resolving set of minimum cardinality.

Note that it is necessary to assume that $|T| \geqslant 3$ in Lemma 2.8 . For example, $\{x, z\}$ is a twin-set of the 3 -vertex path $P_{3}=(x, y, z)$, but $\beta\left(P_{3}\right)=\beta\left(P_{3} \backslash x\right)=1$.

Corollary 2.9. Let $T$ be a twin-set of a connected graph $G$ with $|T| \geqslant 3$. Then $\beta(G)=$ $\beta(G \backslash S)+|S|$ for every subset $S \subset T$ with $|S| \leqslant|T|-2$.

\subsection{The Twin Graph}

Let $G$ be a graph. Define a relation $\equiv$ on $V(G)$ by $u \equiv v$ if and only if $u=v$ or $u, v$ are twins. By Lemma 2.6, $\equiv$ is an equivalence relation. For each vertex $v \in V(G)$, let $v^{*}$ be the set of vertices of $G$ that are equivalent to $v$ under $\equiv$. Let $\left\{v_{1}^{*}, \ldots, v_{k}^{*}\right\}$ be the partition of $V(G)$ induced by $\equiv$, where each $v_{i}$ is a representative of the set $v_{i}^{*}$. The twin graph of $G$, denoted by $G^{*}$, is the graph with vertex set $V\left(G^{*}\right):=\left\{v_{1}^{*}, \ldots, v_{k}^{*}\right\}$, where $v_{i}^{*} v_{j}^{*} \in E\left(G^{*}\right)$ if and only if $v_{i} v_{j} \in E(G)$. The next lemma implies that this definition is independent of the choice of representatives.

Lemma 2.10. Let $G^{*}$ be the twin graph of a graph $G$. Then two vertices $v^{*}$ and $w^{*}$ of $G^{*}$ are adjacent if and only if every vertex in $v^{*}$ is adjacent to every vertex in $w^{*}$ in $G$.

Proof. If every vertex in $v^{*}$ is adjacent to every vertex in $w^{*}$ in $G$, then $v^{*} w^{*} \in E\left(G^{*}\right)$ by definition. For the converse, suppose that $v^{*} w^{*} \in E\left(G^{*}\right)$. Then some $v \in v^{*}$ is adjacent to some $w \in w^{*}$. Let $r \neq v$ be any vertex in $v^{*}$, and let $s \neq w$ be any vertex in $w^{*}$. Since $v$ and $r$ are twins, $r w \in E(G)$ and $r s \in E(G)$. Since $w$ and $s$ are twins, $s v \in E(G)$ and $s r \in E(G)$. That is, every vertex in $v^{*}$ is adjacent to every vertex in $w^{*}$ in $G$. 
Let $N_{r}$ denote the null graph with $r$ vertices and no edges.

Each vertex $v^{*}$ of $G^{*}$ is a maximal twin-set of $G$. By Lemma 2.7, $G\left[v^{*}\right]$ is a complete graph if the vertices of $v^{*}$ are adjacent twins, or $G\left[v^{*}\right]$ is a null graph if the vertices of $v^{*}$ are non-adjacent twins. So it makes sense to consider the following types of vertices in $G^{*}$. We say that $v^{*} \in V\left(G^{*}\right)$ is of type:

- (1) if $\left|v^{*}\right|=1$,

- $(K)$ if $G\left[v^{*}\right] \cong K_{r}$ and $r \geqslant 2$,

- $(N)$ if $G\left[v^{*}\right] \cong N_{r}$ and $r \geqslant 2$.

A vertex of $G^{*}$ is of type $(1 K)$ if it is of type $(1)$ or $(K)$. A vertex of $G^{*}$ is of type $(1 N)$ if it is of type (1) or $(N)$. A vertex of $G^{*}$ is of type $(K N)$ if it is of type $(K)$ or $(N)$.

Observe that the graph $G$ is uniquely determined by $G^{*}$, and the type and cardinality of each vertex of $G^{*}$. In particular, if $v^{*}$ is adjacent to $w^{*}$ in $G^{*}$, then every vertex in $v^{*}$ is adjacent to every vertex in $w^{*}$ in $G$.

We now show that the diameters of $G$ and $G^{*}$ are closely related.

Lemma 2.11. Let $G \neq K_{1}$ be a connected graph. Then $\operatorname{diam}\left(G^{*}\right) \leqslant \operatorname{diam}(G)$. Moreover, $\operatorname{diam}\left(G^{*}\right)<\operatorname{diam}(G)$ if and only if $G^{*} \cong K_{n}$ for some $n \geqslant 1$. In particular, if $\operatorname{diam}(G) \geqslant$ 3 then $\operatorname{diam}(G)=\operatorname{diam}\left(G^{*}\right)$.

Proof. If $v, w$ are adjacent twins in $G$, then $\operatorname{dist}_{G}(v, w)=1$ and $v^{*}=w^{*}$. If $v, w$ are nonadjacent twins in $G$, then (since $G$ has no isolated vertices) $\operatorname{dist}_{G}(v, w)=2$ and $v^{*}=w^{*}$. If $v, w$ are not twins, then there is a shortest path between $v$ and $w$ that contains no pair of twins (otherwise there is a shorter path); thus

$$
\operatorname{dist}_{G}(v, w)=\operatorname{dist}_{G^{*}}\left(v^{*}, w^{*}\right) .
$$

This implies that $\operatorname{diam}\left(G^{*}\right) \leqslant \operatorname{diam}(G)$. Moreover, if $\operatorname{ecc}_{G}(v) \geqslant 3$ then $v$ is not a twin of every vertex $w$ for which $\operatorname{dist}_{G}(v, w)=\operatorname{ecc}_{G}(v)$; thus $\operatorname{dist}_{G}(v, w)=\operatorname{dist}_{G^{*}}\left(v^{*}, w^{*}\right)$ by Equation (1) and $\operatorname{ecc}_{G}(v)=\operatorname{ecc}_{G^{*}}\left(v^{*}\right)$. Hence if $\operatorname{diam}(G) \geqslant 3$ then $\operatorname{diam}(G)=\operatorname{diam}\left(G^{*}\right)$.

Now suppose that $\operatorname{diam}(G)>\operatorname{diam}\left(G^{*}\right)$. Thus $\operatorname{diam}(G) \leqslant 2$. If $\operatorname{diam}(G)=1$ then $G$ is a complete graph and $G^{*} \cong K_{1}$, as claimed. Otherwise $\operatorname{diam}(G)=2$ and $\operatorname{diam}\left(G^{*}\right) \leqslant 1$; thus $G^{*} \cong K_{n}$ for some $n \geqslant 1$, as claimed.

It remains to prove that $\operatorname{diam}\left(G^{*}\right)<\operatorname{diam}(G)$ whenever $G^{*} \cong K_{n}$. In this case, $\operatorname{diam}\left(G^{*}\right) \leqslant 1$. So we are done if $\operatorname{diam}(G) \geqslant 2$. Otherwise $\operatorname{diam}(G) \leqslant 1$ and $G$ is also a complete graph. Thus $G^{*} \cong K_{1}$ and $\operatorname{diam}\left(G^{*}\right)=0$. Since $G \neq K_{1}$, we have $\operatorname{diam}(G)=1>0=\operatorname{diam}\left(G^{*}\right)$, as desired.

Note that graphs with $\operatorname{diam}\left(G^{*}\right)<\operatorname{diam}(G)$ include the complete multipartite graphs.

Theorem 2.14 below characterises the graphs in $\mathcal{G}_{\beta, D}$ for $D \geqslant 3$ in terms of the twin graph. Chartrand et al. [5] characterised ${ }^{5}$ the graphs in $\mathcal{G}_{\beta, D}$ for $D \leqslant 2$. For consistency

\footnotetext{
${ }^{5}$ To be more precise, Chartrand et al. [5] characterised the graphs with $\beta(G)=n-2$. By Lemma 2.2 , if $\beta(G)=n-2$ then $G$ has diameter at most 2. By Proposition 2.12 if $G$ has diameter 1 then $\beta(G)=n-1$. Thus if $\beta(G)=n-2$ then $G$ has diameter 2 .
} 
with Theorem 2.14, we describe the characterisation by Chartrand et al. [5] in terms of the twin graph.

Proposition $2.12([5])$. The following are equivalent for a connected graph $G$ with $n \geqslant 2$ vertices:

- $G$ has metric dimension $\beta(G)=n-1$,

- $G \cong K_{n}$,

- $\operatorname{diam}(G)=1$,

- the twin graph $G^{*}$ has one vertex, which is of type $(K)$.

Proposition $2.13([5])$. The following are equivalent for a connected graph $G$ with $n \geqslant 3$ vertices:

- $G$ has metric dimension $\beta(G)=n-2$,

- $G$ has metric dimension $\beta(G)=n-2$ and diameter $\operatorname{diam}(G)=2$,

- the twin graph $G^{*}$ of $G$ satisfies

$-G^{*} \cong P_{2}$ with at least one vertex of type $(N)$, or

- $G^{*} \cong P_{3}$ with one leaf of type (1), the other leaf of type $(1 K)$, and the degree-2 vertex of type $(1 K)$.

To describe our characterisation we introduce the following notation. Let $P_{D+1}=$ $\left(u_{0}, u_{1}, \ldots, u_{D}\right)$ be a path of length $D$. As illustrated in Figure 11(a), for $k \in[3, D-1]$ let $P_{D+1, k}$ be the graph obtained from $P_{D+1}$ by adding one vertex adjacent to $u_{k-1}$. As illustrated in Figure 1(b), for $k \in[2, D-1]$ let $P_{D+1, k}^{\prime}$ be the graph obtained from $P_{D+1}$ by adding one vertex adjacent to $u_{k-1}$ and $u_{k}$.

(a)

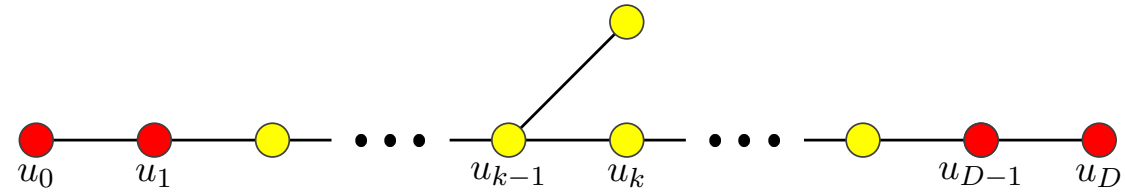

(b)

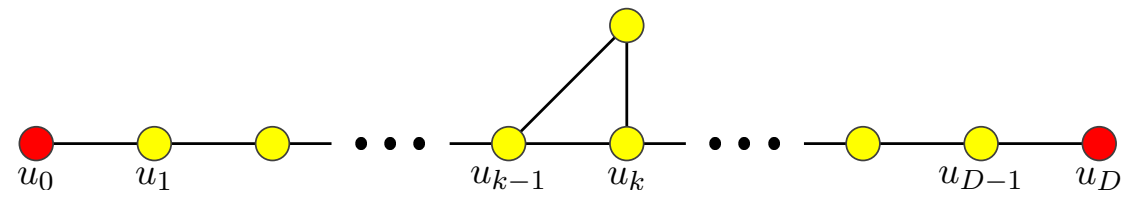

Figure 1: The graphs (a) $P_{D+1, k}$ and (b) $P_{D+1, k}^{\prime}$. 
Theorem 2.14. Let $G$ be a connected graph of order $n$ and diameter $D \geqslant 3$. Let $G^{*}$ be the twin graph of $G$. Let $\alpha\left(G^{*}\right)$ be the number of vertices of $G^{*}$ of type $(K)$ or $(N)$. Then $\beta(G)=n-D$ if and only if $G^{*}$ is one of the following graphs:

1. $G^{*} \cong P_{D+1}$ and one of the following cases holds (see Figure 2):

(a) $\alpha\left(G^{*}\right) \leqslant 1$;

(b) $\alpha\left(G^{*}\right)=2$, the two vertices of $G^{*}$ not of type (1) are adjacent, and if one is a leaf of type $(K)$ then the other is also of type $(K)$;

(c) $\alpha\left(G^{*}\right)=2$, the two vertices of $G^{*}$ not of type (1) are at distance 2 and both are of type $(N)$; or

(d) $\alpha\left(G^{*}\right)=3$ and there is a vertex of type $(N)$ or $(K)$ adjacent to two vertices of type $(N)$.

2. $G^{*} \cong P_{D+1, k}$ for some $k \in[3, D-1]$, the degree-3 vertex $u_{k-1}^{*}$ of $G^{*}$ is any type, each neighbour of $u_{k-1}^{*}$ is type $(1 N)$, and every other vertex is type (1); see Figure 3 .

3. $G^{*} \cong P_{D+1, k}^{\prime}$ for some $k \in[2, D-1]$, the three vertices in the cycle are of type $(1 K)$, and every other vertex is of type (1); see Figure 4 .

(a)

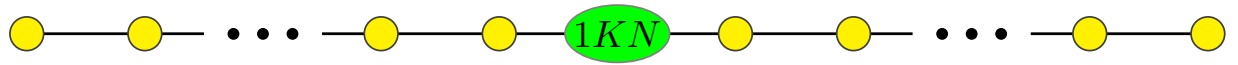

(b)

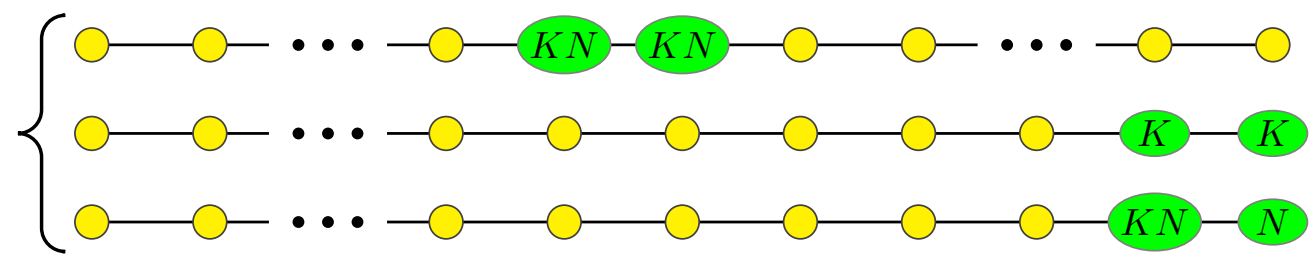

(c)

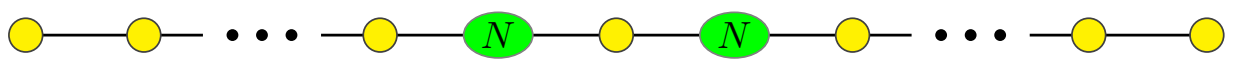

(d)

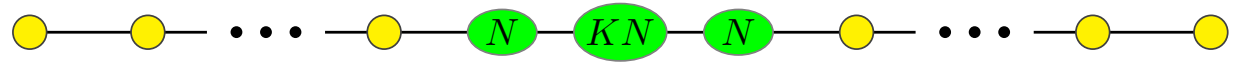

Figure 2: Cases (a)-(d) with $G^{*} \cong P_{D+1}$ in Theorem 2.14.

\subsection{Proof of Necessity}

Throughout this section, $G$ is a connected graph of order $n$, diameter $D \geqslant 3$, and metric dimension $\beta(G)=n-D$. Let $G^{*}$ be the twin graph of $G$.

Lemma 2.15. There exists a vertex $u_{0}$ in $G$ of eccentricity $D$ with no twin. 


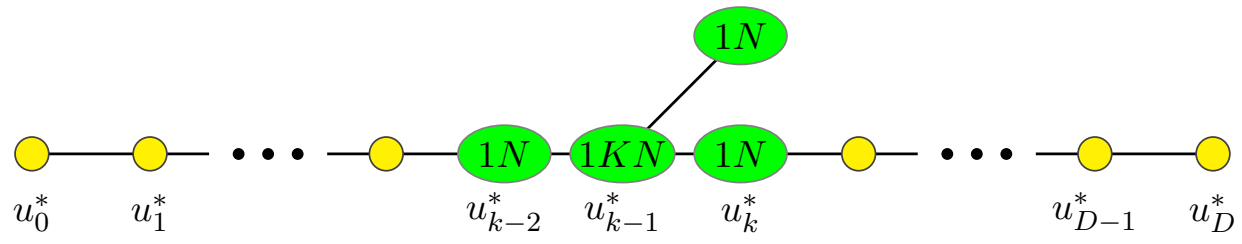

Figure 3: The case of $G^{*} \cong P_{D+1, k}$ in Theorem 2.14

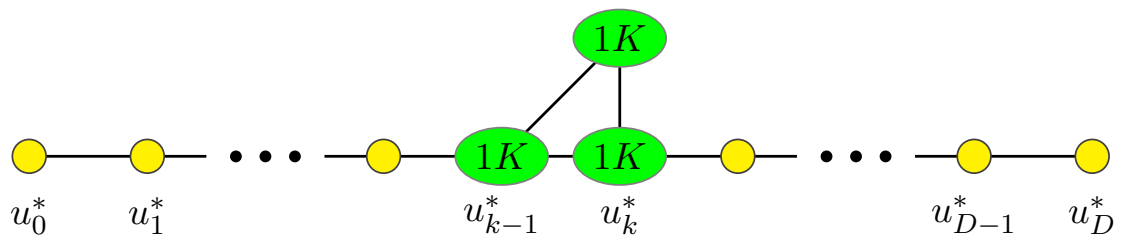

Figure 4: The case of $G^{*} \cong P_{D+1, k}^{\prime}$ in Theorem 2.14

Proof. Let $u_{0}$ and $u_{D}$ be vertices at distance $D$ in $G$. As illustrated in Figure 5, let $\left(u_{0}, u_{1}, \ldots, u_{D}\right)$ be a shortest path between $u_{0}$ and $u_{D}$. Suppose on the contrary that both $u_{0}$ and $u_{D}$ have twins. Let $x$ be a twin of $u_{0}$ and $y$ be a twin of $u_{D}$. We claim that $\{x, y\}$ resolves $\left\{u_{0}, \ldots, u_{D}\right\}$. Now $u_{0} \not u_{i}$ for all $i \in[2, D]$, and thus $x \not u_{i}$ (since $x, u_{0}$ are twins). Thus $\operatorname{dist}\left(x, u_{i}\right)=i$ for each $i \in[1, D]$. Hence $x$ resolves $u_{i}, u_{j}$ for all distinct $i, j \in[1, D]$. By symmetry, $\operatorname{dist}\left(y, u_{i}\right)=D-i$ for all $i \in[0, D-1]$, and $y$ resolves $u_{i}, u_{j}$ for all distinct $i, j \in[0, D-1]$. Thus $\{x, y\}$ resolves $\left\{u_{0}, \ldots, u_{D}\right\}$, except for possibly the pair $u_{0}, u_{D}$. Now $\operatorname{dist}\left(x, u_{0}\right) \leqslant 2$ and $\operatorname{dist}\left(x, u_{D}\right)=D$. Since $D \geqslant 3, x$ resolves $u_{0}, u_{D}$. Thus $\{x, y\}$ resolves $\left\{u_{0}, \ldots, u_{D}\right\}$. By Lemma 2.1, $\beta(G) \leqslant n-(D+1)<n-D$, which is a contradiction. Thus $u_{0}$ or $u_{D}$ has no twin.

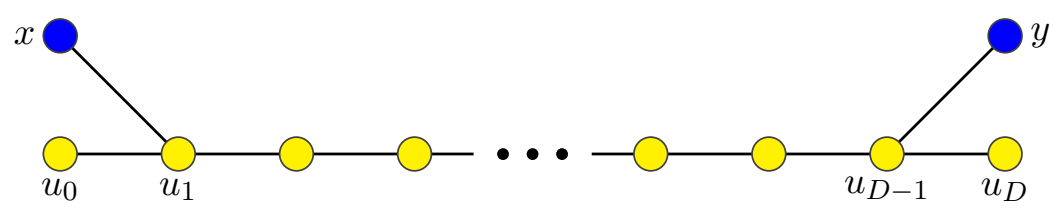

Figure 5: $\{x, y\}$ resolves $\left\{u_{0}, \ldots, u_{D}\right\}$ in Lemma 2.15.

For the rest of the proof, fix a vertex $u_{0}$ of eccentricity $D$ in $G$ with no twin, which exists by Lemma 2.15. Thus $u_{0}^{*}=\left\{u_{0}\right\}$ and $\operatorname{ecc}_{G^{*}}\left(u_{0}^{*}\right)=\operatorname{ecc}_{G}\left(u_{0}\right)=D$, which is also the diameter of $G^{*}$ by Lemma 2.11. As illustrated in Figure 6, for each $i \in[0, D]$, let

$$
\begin{aligned}
& A_{i}^{*}:=\left\{v^{*} \in V\left(G^{*}\right): \operatorname{dist}\left(u_{0}^{*}, v^{*}\right)=i\right\}, \text { and } \\
& A_{i}:=\left\{v \in V(G): \operatorname{dist}\left(u_{0}, v\right)=i\right\}=\bigcup\left\{v^{*}: v^{*} \in A_{i}^{*}\right\} .
\end{aligned}
$$


Note that the last equality is true because $u_{0}$ has no twin and $\operatorname{dist}\left(u_{0}, v\right)=\operatorname{dist}\left(u_{0}, w\right)$ if $v, w$ are twins. For all $i \in[0, D]$, we have $\left|A_{i}\right| \geqslant 1$ and $\left|A_{i}^{*}\right| \geqslant 1$. Moreover, $\left|A_{0}\right|=\left|A_{0}^{*}\right|=$ 1. Let $\left(u_{0}, u_{1}, \ldots, u_{D}\right)$ be a path in $G$ such that $u_{i} \in A_{i}$ for each $i \in[0, D]$. Observe that if $v \in A_{i}$ is adjacent to $w \in A_{j}$ then $|i-j| \leqslant 1$. In particular, $\left(u_{i}, u_{i+1}, \ldots, u_{j}\right)$ is a shortest path between $u_{i}$ and $u_{j}$.

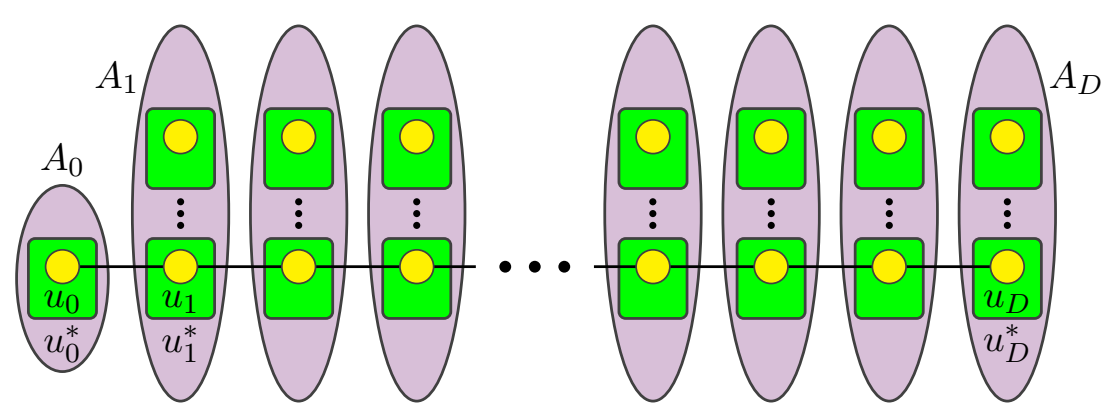

Figure 6: The sets $A_{0}, A_{1}, \ldots, A_{D}$.

Lemma 2.16. For each $k \in[1, D]$,

- $G\left[A_{k}\right]$ is a complete graph or a null graph;

- $G^{*}\left[A_{k}^{*}\right]$ is a complete graph or a null graph, and all the vertices in $A_{k}^{*}$ are of type $(1 K)$ in the first case, and of type $(1 N)$ in the second case.

Proof. Suppose that $G\left[A_{k}\right]$ is neither complete nor null for some $k \in[1, D]$. Thus there exist vertices $u, v, w \in A_{k}$ such that $u \sim v \nsim w$, as illustrated in Figure 抑. Let $S:=$ $\left(\left\{u_{1}, \ldots, u_{D}\right\} \backslash\left\{u_{k}\right\}\right) \cup\{u, w\}$. Every pair of vertices in $S$ is resolved by $u_{0}$, except for $u, w$, which is resolved by $v$. Thus $\left\{u_{0}, v\right\}$ resolves $S$. By Lemma $2.1, \beta(G) \leqslant n-(D+1)<$ $n-D$. This contradiction proves the first claim, which immediately implies the second claim.

Lemma 2.17. For each $k \in[1, D]$, if $\left|A_{k}\right| \geqslant 2$ then

(a) $v \sim w$ for all vertices $v \in A_{k-1}$ and $w \in A_{k}$;

(b) $v^{*} \sim w^{*}$ for all vertices $v^{*} \in A_{k-1}^{*}$ and $w^{*} \in A_{k}^{*}$.

Proof. First we prove (a). Every vertex in $A_{1}$ is adjacent to $u_{0}$, which is the only vertex in $A_{0}$. Thus (a) is true for $k=1$. Now assume that $k \geqslant 2$. Suppose on the contrary that $v \nsim w$ for some $v \in A_{k-1}$ and $w \in A_{k}$. There exists a vertex $u \in A_{k-1}$ adjacent to $w$. As illustrated in Figure 8, if $w \neq u_{k}$ then $\left\{u_{0}, w\right\}$ resolves $\left(\left\{u_{1}, \ldots, u_{D}\right\} \backslash\left\{u_{k-1}\right\}\right) \cup\{u, v\}$.

\footnotetext{
${ }^{6}$ In Figures $7[22$, a solid line connects adjacent vertices, a dashed line connects non-adjacent vertices, and a coil connects vertices that may or may not be adjacent.
} 


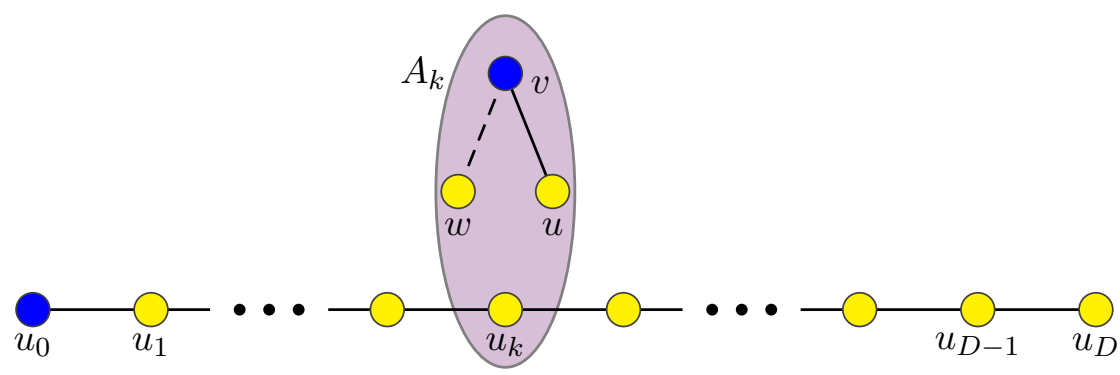

Figure 7: $\left\{u_{0}, v\right\}$ resolves $\left(\left\{u_{1}, \ldots, u_{D}\right\} \backslash\left\{u_{k}\right\}\right) \cup\{u, w\}$ in Lemma 2.16.

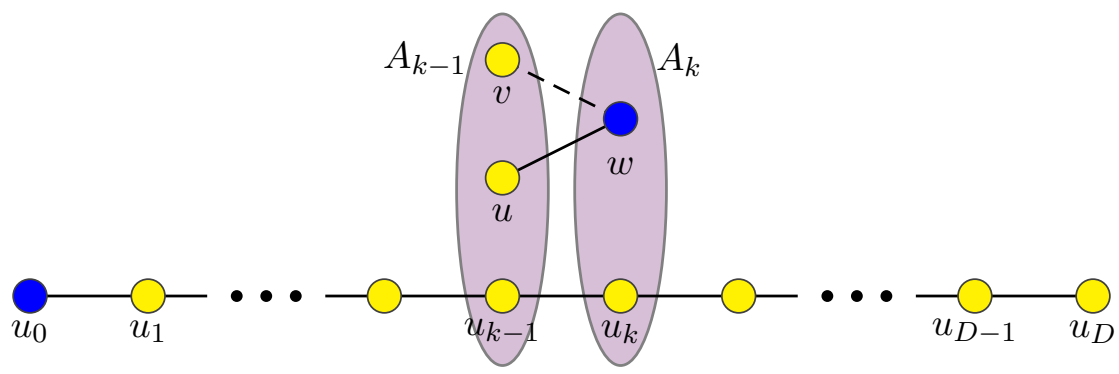

Figure 8: In Lemma 2.17, $\left\{u_{0}, w\right\}$ resolves $\left(\left\{u_{1}, \ldots, u_{D}\right\} \backslash\left\{u_{k-1}\right\}\right) \cup\{u, v\}$.

As illustrated in Figure 9, if $w=u_{k}$ then $v \neq u_{k-1}$ and there exists a vertex $z \neq u_{k}$ in $A_{k}$, implying $\left\{u_{0}, u_{k}\right\}$ resolves $\left(\left\{u_{1}, \ldots, u_{D}\right\} \backslash\left\{u_{k}\right\}\right) \cup\{v, z\}$. In both cases, Lemma 2.1 implies that $\beta(G) \leqslant n-D-1$. This contradiction proves (a), which immediately implies (b).

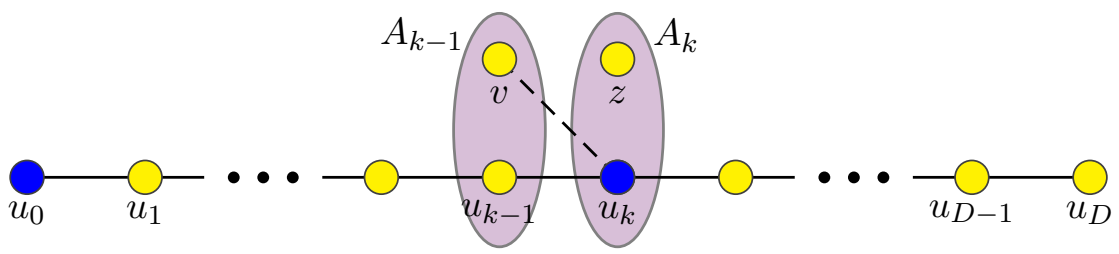

Figure 9: In Lemma 2.17, $\left\{u_{0}, u_{k}\right\}$ resolves $\left(\left\{u_{1}, \ldots, u_{D}\right\} \backslash\left\{u_{k}\right\}\right) \cup\{v, z\}$.

Lemma 2.18. If $\left|A_{i}\right| \geqslant 2$ and $\left|A_{j}\right| \geqslant 2$ then $|i-j| \leqslant 2$. Thus there are at most three distinct subsets $A_{i}, A_{j}, A_{k}$ each with cardinality at least 2.

Proof. Suppose on the contrary that $\left|A_{i}\right| \geqslant 2$ and $\left|A_{j}\right| \geqslant 2$ for some $i, j \in[1, D]$ with $j \geqslant i+3$, as illustrated in Figure 10 . Let $x \neq u_{i}$ be a vertex in $A_{i}$. Let $y \neq u_{j}$ be a vertex in $A_{j}$. We claim that $\left\{u_{j}, x\right\}$ resolves $\left(\left\{u_{0}, \ldots, u_{D}\right\} \backslash\left\{u_{j}\right\}\right) \cup\{y\}$. 
By Lemma 2.17, $u_{i-1} \sim x$ and $u_{j-1} \sim y$. Observe that $\operatorname{dist}\left(u_{j}, y\right) \in\{1,2\} ; \operatorname{dist}\left(u_{j}, u_{j-h}\right)=$ $h$ for all $h \in[1, j] ; \operatorname{dist}\left(u_{j}, u_{j+h}\right)=h$ for all $h \in[1, D-j]$. Thus $u_{j}$ resolves $\left(\left\{u_{0}, \ldots, u_{D}\right\} \backslash\right.$ $\left.\left\{u_{j}\right\}\right) \cup\{y\}$, except for the following pairs:

- $u_{j-h}, u_{j+h}$ whenever $1 \leqslant h \leqslant j \leqslant D-h$;

- $y, u_{j-1}$ and $y, u_{j+1}$ if $\operatorname{dist}\left(y, u_{j}\right)=1$; and

- $y, u_{j-2}$ and $y, u_{j+2}$ if $\operatorname{dist}\left(y, u_{j}\right)=2$.

We claim that $x$ resolves each of these pairs. By Lemma 2.17, there is a shortest path between $x$ and $u_{j-1}$ that passes through $u_{j-2}$. Let $r:=\operatorname{dist}\left(x, u_{j-2}\right)$. Thus $\operatorname{dist}\left(x, u_{j-1}\right)=$ $r+1, \operatorname{dist}(x, y)=r+2, \operatorname{dist}\left(x, u_{j+1}\right)=r+3$, and $\operatorname{dist}\left(x, u_{j+2}\right)=r+4$. Thus $x$ resolves every pair of vertices in $\left\{u_{j-2}, u_{j-1}, y, u_{j+1}, u_{j+2}\right\}$. It remains to prove that $x$ resolves $u_{j-h}, u_{j+h}$ whenever $3 \leqslant h \leqslant j \leqslant D-h$. Observe that $\operatorname{dist}\left(x, u_{j+h}\right) \geqslant j+h-i$. If $j-h \geqslant i$ then, since $\left(x, u_{i-1}, \ldots, u_{j-h}\right)$ is a path,

$$
\operatorname{dist}\left(x, u_{j-h}\right) \leqslant j-h-i+2<j+h-i \leqslant \operatorname{dist}\left(x, u_{j+h}\right) .
$$

Otherwise $j-h \leqslant i-1$, implying

$$
\operatorname{dist}\left(x, u_{j-h}\right)=i-(j-h)<j+h-i \leqslant \operatorname{dist}\left(x, u_{j+h}\right) .
$$

In each case $\operatorname{dist}\left(x, u_{j-h}\right)<\operatorname{dist}\left(x, u_{j+h}\right)$. Thus $x$ resolves $u_{j-h}, u_{j+h}$.

Hence $\left\{u_{j}, x\right\}$ resolves $\left(\left\{u_{0}, \ldots, u_{D}\right\} \backslash\left\{u_{j}\right\}\right) \cup\{y\}$. By Lemma 2.1, $\beta(G) \leqslant n-D-1$ which is the desired contradiction.

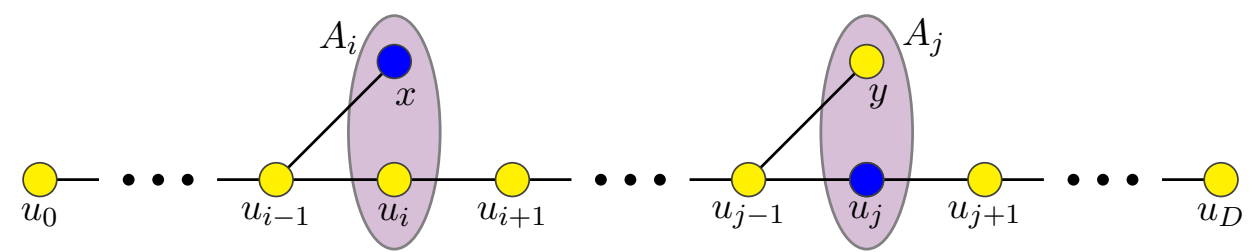

Figure 10: $\left\{u_{j}, x\right\}$ resolves $\left(\left\{u_{0}, \ldots, u_{D}\right\} \backslash\left\{u_{j}\right\}\right) \cup\{y\}$ in Lemma 2.18.

Lemma 2.19. $\left|A_{1}^{*}\right|=1$ and $\left|A_{D}^{*}\right|=1$.

Proof. Consider a vertex $v \in A_{1}$. Then $v \sim u_{0}$ and every other neighbour of $v$ is in $A_{1} \cup A_{2}$. By Lemma 2.16, $G\left[A_{1}\right]$ is complete or null. If every vertex in $A_{1}$ is adjacent to every vertex in $A_{2}$, then $A_{1}$ is a twin-set, and $\left|A_{1}^{*}\right|=1$ as desired.

Now assume that some vertex $v \in A_{1}$ is not adjacent to some vertex in $A_{2}$. By Lemma 2.17, the only vertex in $A_{2}$ is $u_{2}$, and $v \not u_{2}$. If $G\left[A_{1}\right]$ is null then $\operatorname{ecc}(v)>D$, and if $G\left[A_{1}\right]$ is complete then $v$ and $u_{0}$ are twins. In both cases we have a contradiction.

If $\left|A_{D}\right|=1$ then $\left|A_{D}^{*}\right|=1$. Now assume that $\left|A_{D}\right| \geqslant 2$. The neighbourhood of every vertex in $A_{D}$ is contained in $A_{D-1} \cup A_{D}$. By Lemma 2.17, every vertex in $A_{D}$ is adjacent to every vertex in $A_{D-1}$. By Lemma 2.16, $G\left[A_{D}\right]$ is complete or null. Thus $A_{D}$ is a twin-set, implying $\left|A_{D}^{*}\right|=1$. 
Lemma 2.20. For each $k \in[1, D-1]$, distinct vertices $v, w \in A_{k}$ are twins if and only if they have the same neighbourhood in $A_{k+1}$.

Proof. The neighbourhood of both $v$ and $w$ is contained in $A_{k-1} \cup A_{k} \cup A_{k+1}$. By Lemma 2.17, both $v$ and $w$ are adjacent to every vertex in $A_{k-1}$. By Lemma 2.16, $G\left[A_{k}\right]$ is complete or null. Thus $v$ and $w$ are twins if and only if they have the same neighbourhood in $A_{k+1}$.

Lemma 2.21. For each $k \in[2, D]$,

(a) if $\left|A_{k}\right| \geqslant 2$ then $\left|A_{k-1}^{*}\right|=1$;

(b) if $\left|A_{k}\right|=1$ then $\left|A_{k-1}^{*}\right| \leqslant 2$.

Proof. Suppose that $\left|A_{k}\right| \geqslant 2$. If $\left|A_{k-1}\right|=1$ then $\left|A_{k-1}^{*}\right|=1$ as desired. Now assume that $\left|A_{k-1}\right| \geqslant 2$. Thus $A_{k-1}$ is a twin-set by Lemmas 2.17 and 2.20 , implying $\left|A_{k-1}^{*}\right|=1$. Now suppose that $\left|A_{k}\right|=1$. If $\left|A_{k-1}\right|=1$ then $\left|A_{k-1}^{*}\right|=1$ and we are done. So assume that $\left|A_{k-1}\right| \geqslant 2$. By Lemma 2.20 , the set of vertices in $A_{k-1}$ that are adjacent to the unique vertex in $A_{k}$ is a maximal twin-set, and the set of vertices in $A_{k-1}$ that are not adjacent to the unique vertex in $A_{k}$ is a maximal twin-set (if it is not empty). Therefore $\left|A_{k-1}^{*}\right| \leqslant 2$.

Lemma 2.22. For each $k \in[1, D]$, we have $\left|A_{k}^{*}\right| \leqslant 2$. Moreover, there are at most three values of $k$ for which $\left|A_{k}^{*}\right|=2$. Furthermore, if $\left|A_{i}^{*}\right|=2$ and $\left|A_{j}^{*}\right|=2$ then $|i-j| \leqslant 2$.

Proof. Lemma 2.19 proves the result for $k=D$. Now assume that $k \in[1, D-1]$. Suppose on the contrary that $\left|A_{k}^{*}\right| \geqslant 3$ for some $k \in[1, D]$. By the contrapositive of Lemma 2.21(a), $\left|A_{k+1}\right|=1$. By Lemma 2.21(b), $\left|A_{k}^{*}\right| \leqslant 2$, which is the desired contradiction. The remaining claims follow immediately from Lemma 2.18 .

Lemma 2.23. Suppose that $\left|A_{k}^{*}\right|=2$ for some $k \in[2, D-1]$. Then $\left|A_{k+1}\right|=\left|A_{k+1}^{*}\right|=1$, and exactly one of the two vertices of $A_{k}^{*}$ is adjacent to the only vertex of $A_{k+1}^{*}$. Moreover, if $k \leqslant D-2$ then $\left|A_{k+2}\right|=\left|A_{k+2}^{*}\right|=1$.

Proof. By the contrapositive of Lemma 2.21(a), $\left|A_{k+1}\right|=\left|A_{k+1}^{*}\right|=1$. By Lemma 2.20 . exactly one vertex in $A_{k}^{*}$ is adjacent to the vertex in $A_{k+1}^{*}$. Now suppose that $k \leqslant D-2$ but $\left|A_{k+2}\right| \geqslant 2$. As illustrated in Figure 11, let $x \neq u_{k+2}$ be a vertex in $A_{k+2}$. Let $y \neq u_{k}$ be a vertex in $A_{k}$, such that $y, u_{k}$ are not twins, that is, $y \nsim u_{k+1}$. By Lemma 2.17, $u_{k-1} \sim y$ and $u_{k+1} \sim x$. Thus $\left\{x, u_{0}\right\}$ resolves $\left\{u_{1}, \ldots, u_{D}, y\right\}$. By Lemma 2.1, $\beta(G) \leqslant n-D-1$, which is a contradiction. Hence $\left|A_{k+2}\right|=1$, implying $\left|A_{k+2}^{*}\right|=1$.

We now prove that the structure of the graph $G^{*}$ is as claimed in Theorem 2.14.

Lemma 2.24. Either $G^{*} \cong P_{D+1}, G^{*} \cong P_{D+1, k}$ for some $k \in[3, D-1]$, or $G^{*} \cong P_{D+1, k}^{\prime}$ for some $k \in[2, D-1]$. 


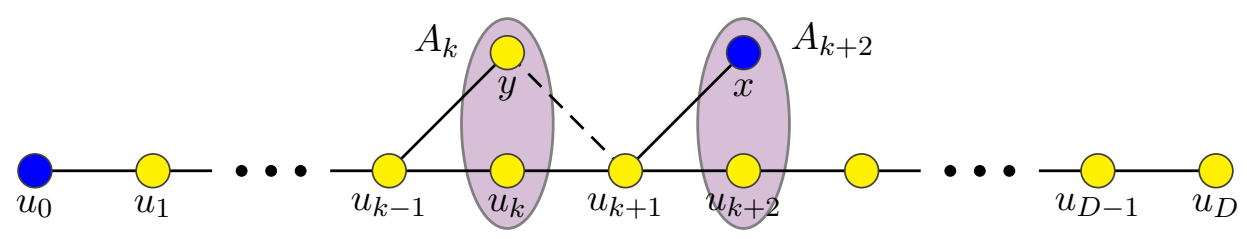

Figure 11: $\left\{x, u_{0}\right\}$ resolves $\left\{u_{1}, \ldots, u_{D}, y\right\}$ in Lemma 2.23 .

Proof. By Lemma 2.22 each set $A_{k}^{*}$ contains at most two vertices of $G^{*}$. Lemmas 2.19. 2.18 and 2.23 imply that $\left|A_{k}^{*}\right|=2$ for at most one $k \in[0, D]$. If $\left|A_{k}^{*}\right|=1$ for every $k \in[0, D]$ then $G^{*} \cong P_{D+1}$ as desired.

Now assume that $\left|A_{k}^{*}\right|=2$ for exactly one $k \in[0, D]$. By Lemma 2.19, $k \in[2, D-1]$. Let $w^{*}$ be the vertex in $A_{k}^{*}$ besides $u_{k}^{*}$. Then $w^{*} \sim u_{k-1}^{*}$ by Lemma 2.17. If $w^{*} \sim u_{k}^{*}$ then $G^{*} \cong P_{D+1, k}^{\prime}$. Otherwise $w^{*} \nsim u_{k}^{*}$. Then $G^{*} \cong P_{D+1, k}$. It remains to prove that in this case $k \neq 2$.

Suppose on the contrary that $G^{*} \cong P_{D+1, k}$ and $k=2$. Thus $\left|A_{2}^{*}\right|=2$. Say $A_{2}^{*}=$ $\left\{u_{2}^{*}, w^{*}\right\}$, where $u_{2}^{*} \nsim w^{*}$. By Lemma 2.23, $\left|A_{3}^{*}\right|=1$. Thus $A_{3}^{*}=\left\{u_{3}^{*}\right\}$. Since $u_{2}^{*} \sim u_{3}^{*}$, by Lemma 2.20, $w^{*} \chi u_{3}^{*}$. Thus $u_{1}^{*}$ is the only neighbour of $w^{*}$. Hence every vertex in $w^{*}$ is a twin of $u_{0}$, which contradicts the fact that $u_{0}$ has no twin. Thus $k \neq 2$ if $G^{*} \cong P_{D+1, k}$.

We now prove restrictions about the type of vertices in $G^{*}$. To start with, Lemma 2.18 implies:

Corollary 2.25. If $G^{*} \cong P_{D+1}$ then $\alpha\left(G^{*}\right) \leqslant 3$ and the distance between every pair of vertices not of type (1) is at most 2.

Lemma 2.26. Suppose that $G^{*} \cong P_{D+1}$ and $\alpha\left(G^{*}\right)=2$. If the two vertices of $G^{*}$ not of type (1) are adjacent, and one of them is a leaf of type $(K)$, then the other is also of type $(K)$.

Proof. As illustrated in Figure 12, let $x$ and $y$ be twins of $u_{D-1}$ and $u_{D}$ respectively. By assumption $G\left[A_{D}\right]$ is a complete graph. Suppose on the contrary that $G\left[A_{D-1}\right]$ is a null graph. By Lemma 2.17, every vertex in $A_{D}$ is adjacent to every vertex in $A_{D-1}$. Thus $y$ resolves $\left\{u_{0}, \ldots, u_{D}\right\}$, except for the pair $u_{D-1}, u_{D}$, which is resolved by $x$. Thus $\{x, y\}$ resolves $\left\{u_{0}, \ldots, u_{D}\right\}$. By Lemma 2.1, $\beta(G) \leqslant n-D-1$, which is a contradiction. Thus $G\left[A_{D-1}\right]$ is a complete graph.

Lemma 2.27. Suppose that $G^{*} \cong P_{D+1}$ and for some $k \in[2, D-1]$, the vertices $u_{k-1}^{*}$ and $u_{k+1}^{*}$ of $G^{*}$ are both not of type (1). Then $u_{k-1}^{*}$ and $u_{k+1}^{*}$ are both of type $(N)$.

Proof. Let $x$ and $y$ be twins of $u_{k-1}$ and $u_{k+1}$ respectively. Suppose on the contrary that one of $u_{k-1}^{*}$ and $u_{k+1}^{*}$ is of type $(K)$. Without loss of generality $u_{k-1}^{*}$ is of type $(K)$, as illustrated in Figure 13. Thus $u_{k-1} \sim x$. We claim that $\{x, y\}$ resolves $\left\{u_{0}, u_{1}, \ldots, u_{D}\right\}$.

Observe that $x$ resolves every pair of vertices of $\left\{u_{0}, u_{1}, \ldots, u_{D}\right\}$ except for: 


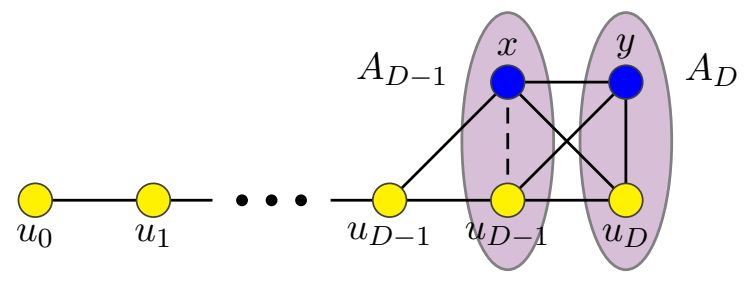

Figure 12: $\{x, y\}$ resolves $\left\{u_{0}, \ldots, u_{D}\right\}$ in Lemma 2.26 .

- each pair of vertices in $\left\{u_{k-2}, u_{k-1}, u_{k}\right\}$, which are all resolved by $y$ since $d\left(y, u_{k}\right)=1$, $d\left(y, u_{k-1}\right)=2$, and $d\left(y, u_{k-2}\right)=3$; and

- the pairs $\left\{u_{k-j}, u_{k+j-2}: j \in[3, \min \{k, D+2-k\}]\right\}$, which are all resolved by $y$ since $d\left(y, u_{k-j}\right)=j+1$, and

$$
d\left(y, u_{k+j-2}\right)= \begin{cases}j-2 & \text { if } j \geqslant 4 \\ 1 \text { or } 2 & \text { if } j=3\end{cases}
$$

Hence $\{x, y\}$ resolves $\left\{u_{0}, u_{1}, \ldots, u_{D}\right\}$. Thus Lemma 2.1 implies $\beta(G) \leqslant n-D-1$, which is the desired contradiction. Hence $u_{k-1}^{*}$ and $u_{k+1}^{*}$ are both of type $(N)$.

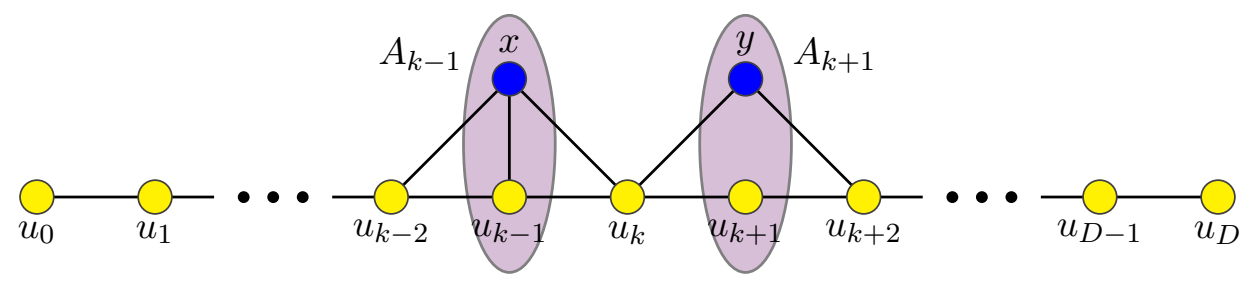

Figure 13: $\{x, y\}$ resolves $\left\{u_{0}, \ldots, u_{D}\right\}$ in Lemma 2.27.

Corollary 2.25 and Lemmas 2.26 and 2.27 prove the necessity of the conditions in Theorem 2.14 when $G^{*} \cong P_{D+1}$.

Lemma 2.28. Suppose that $G^{*} \cong P_{D+1, k}$ for some $k \in[3, D-1]$, where $A_{k}^{*}=\left\{u_{k}^{*}, w^{*}\right\}$ and $w^{*} \sim u_{k-1}^{*}$. Then $u_{k-2}^{*}, u_{k}^{*}$ and $w^{*}$ are type $(1 N), u_{k-1}^{*}$ is any type, and every other vertex is type (1).

Proof. Since $u_{k}^{*} \nsim w^{*}$, Lemma 2.16 implies that $u_{k}^{*}$ and $w^{*}$ are both type $(1 N)$. By Lemmas 2.18 and 2.23 , the remaining vertices are of type (1) except, possibly $u_{k-2}^{*}$ and $u_{k-1}^{*}$. Suppose that $u_{k-2}^{*}$ is of type $(K)$, as illustrated in Figure 14. Let $x$ be a twin of $u_{k-2}$. Then $x \sim u_{k-1}$. We claim that $\{x, w\}$ resolves $\left\{u_{0}, u_{1}, \ldots, u_{D}\right\}$.

Observe that $x$ resolves every pair of vertices in $\left\{u_{0}, u_{1}, \ldots, u_{D}\right\}$ except for: 
- each pair of vertices in $\left\{u_{k-3}, u_{k-2}, u_{k-1}\right\}$, which are all resolved by $w$ since $d\left(w, u_{k-1}\right)=$ $1, d\left(w, u_{k-2}\right)=2$, and $d\left(w, u_{k-3}\right)=3$; and

- the pairs $\left\{u_{k-2-j}, u_{k-2+j}: j \in[2, \min \{k-2, D-k+2\}]\right\}$, which are all resolved by $w$ since $d\left(w, u_{k-2-j}\right)=j+2$ and $d\left(w, u_{k-2+j}\right)=j$.

Thus $\{x, w\}$ resolves $\left\{u_{0}, u_{1}, \ldots, u_{D}\right\}$. Hence Lemma 2.1 implies that $\beta(G) \leqslant n-D-1$, which is the desired contradiction.

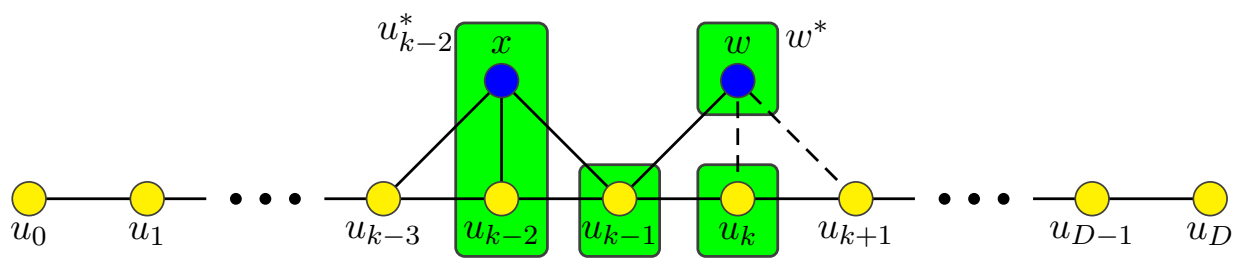

Figure 14: $\{x, w\}$ resolves $\left\{u_{0}, \ldots, u_{D}\right\}$ in Lemma 2.28 .

Lemma 2.29. Suppose that $G^{*} \cong P_{D+1, k}^{\prime}$ for some $k \in[2, D-1]$, where $A_{k}^{*}=\left\{u_{k}^{*}, w^{*}\right\}$ and $u_{k-1}^{*} \sim w^{*} \sim u_{k}^{*}$. Then $u_{k-1}^{*}, u_{k}^{*}$ and $w^{*}$ are type $(1 K)$, and every other vertex is type $(1)$.

Proof. Since $u_{k}^{*} \sim w^{*}$, Lemma 2.16 implies that $u_{k}^{*}$ and $w^{*}$ are type $(1 K)$. By Lemmas 2.18 and 2.23, the remaining vertices are type (1) except possibly $u_{k-2}^{*}$ and $u_{k-1}^{*}$.

Suppose on the contrary that $u_{k-2}^{*}$ is type $(K)$ or $(N)$, as illustrated in Figure 15 . Let $x$ be a twin of $u_{k-2}$. We claim that $\{x, w\}$ resolves $\left\{u_{0}, u_{1}, \ldots, u_{D}\right\}$. Observe that $w$ resolves every pair of vertices in $\left\{u_{0}, u_{1}, \ldots, u_{D}\right\}$, except for pairs

$$
\left\{u_{k-1-j}, u_{k+j}: j \in[0, \min \{k-1, D-k\}]\right\} .
$$

These pairs are all resolved by $x$ since $d\left(x, u_{k+j}\right)=j+2$ and

$$
d\left(x, u_{k-1-j}\right)= \begin{cases}j-1 & \text { if } j \geqslant 2 \\ 1 \text { or } 2 & \text { if } j=1 \\ 1 & \text { if } j=0\end{cases}
$$

Thus $\{x, w\}$ resolves $\left\{u_{0}, u_{1}, \ldots, u_{D}\right\}$.

Suppose on the contrary that $u_{k-1}^{*}$ is type $(N)$, as illustrated in Figure 16 . Let $y$ be a twin of $u_{k-1}$. We claim that $\{y, w\}$ resolves $\left\{u_{0}, u_{1}, \ldots, u_{D}\right\}$. Observe that $w$ resolves every pair of vertices in $\left\{u_{0}, u_{1}, \ldots, u_{D}\right\}$, except for pairs

$$
\left\{u_{k-1-j}, u_{k+j}: j \in[0, \min \{k-1, D-k\}]\right\} \text {. }
$$




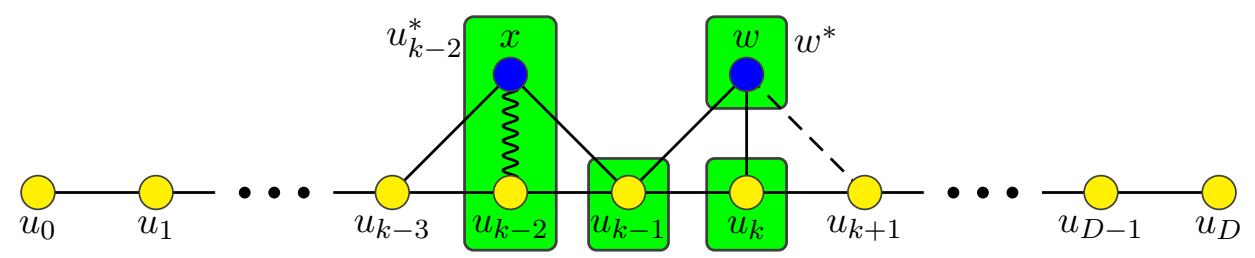

Figure 15: $\{x, w\}$ resolves $\left\{u_{0}, u_{1}, \ldots, u_{D}\right\}$ in Lemma 2.29.

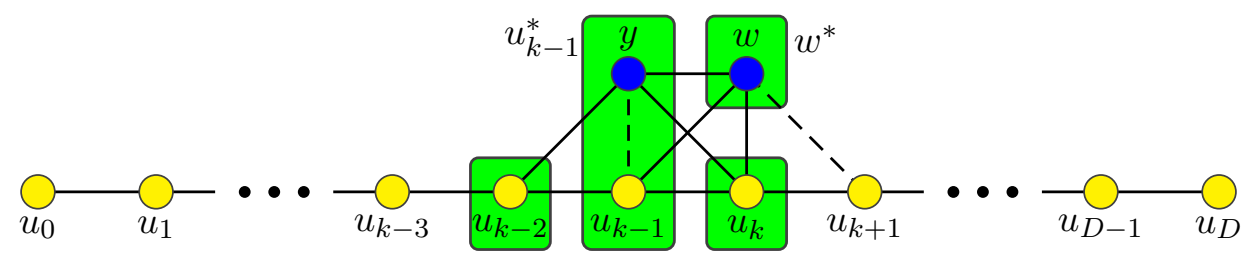

Figure 16: $\{y, w\}$ resolves $\left\{u_{0}, u_{1}, \ldots, u_{D}\right\}$ in Lemma 2.29.

These pairs are all resolved by $y$ since $d\left(y, u_{k+j}\right)=j+1$ and

$$
d\left(y, u_{k-1-j}\right)= \begin{cases}j & \text { if } j \geqslant 1 \\ 2 & \text { if } j=0\end{cases}
$$

Thus $\{y, w\}$ resolves $\left\{u_{0}, u_{1}, \ldots, u_{D}\right\}$.

By Lemma 2.1, in each case $\beta(G) \leqslant n-D-1$, which is the desired contradiction.

Observe that Lemmas 2.28 and 2.29 imply the necessity of the conditions in Theorem 2.14 when $G^{*} \cong P_{D+1, k}$ or $G^{*} \cong P_{D+1, k}^{\prime}$. This completes the proof of the necessity of the conditions in Theorem 2.14 .

\subsection{Proof of Sufficiency}

Let $G$ be a graph with $n$ vertices and $\operatorname{diam}(G) \geqslant 3$. Let $T$ be a twin-set of cardinality $r \geqslant 3$ in $G$. Let $G^{\prime}$ be the graph obtained from $G$ by deleting all but two of the vertices in T. As in Lemma 2.11, $\operatorname{diam}\left(G^{\prime}\right)=\operatorname{diam}(G)$. Say $G^{\prime}$ has order $n^{\prime}$. Then by Corollary 2.9, $\beta\left(G^{\prime}\right)=\beta(G)-(r-2)$. Since $n^{\prime}=n-(r-2)$, we have that $\beta(G)=n-D$ if and only if $\beta\left(G^{\prime}\right)=n^{\prime}-D$. Thus it suffices to prove the sufficiency in Theorem 2.14 for graphs $G$ whose maximal twin-sets have at most two vertices. We assume in the remainder of this section that every twin-set in $G$ has at most two vertices.

Suppose that the twin graph $G^{*}$ of $G$ is one of the graphs stated in Theorem 2.14. We need to prove that $\beta(G)=n-D$. Since $\beta(G) \leqslant n-D$ by Lemma 2.2 , it suffices to prove that every subset of $n-D-1$ vertices of $G$ is not a resolving set. By Corollary 2.4. every resolving set contains at least one vertex in each twin-set of cardinality 2. Observe also that, since $\alpha\left(G^{*}\right)$ is the number of vertices of $G^{*}$ not of type (1), we have that $\alpha\left(G^{*}\right)=n-\left|V\left(G^{*}\right)\right|$. 
Case 1. $G^{*} \cong P_{D+1}$ with vertices $u_{0}^{*} \sim u_{1}^{*} \sim \cdots \sim u_{D}^{*}$ : We now prove that for each subcase stated in Theorem 2.14 every set of $n-D-1=n-\left|V\left(G^{*}\right)\right|=\alpha\left(G^{*}\right)$ vertices of $G$ does not resolve $G$. Suppose on the contrary that $W$ is a resolving set of $G$ of cardinality $\alpha\left(G^{*}\right)$.

Case 1(a). $\alpha\left(G^{*}\right) \leqslant 1$ : We need at least one vertex to resolve a graph $G$ of order $n \geqslant 2$. So $\alpha\left(G^{*}\right)=1$. Thus $G$ is not a path, but Khuller et al. [21] and Chartrand et al. [5] independently proved that every graph with metric dimension 1 is a path, which is a contradiction.

Case 1(b)(i). $\alpha\left(G^{*}\right)=2$, and $u_{k}^{*}, u_{k+1}^{*}$ are not of type (1) for some $k \in[1, D-2]$ : As illustrated in Figure 17, consider vertices $x \neq u_{k}$ in $u_{k}^{*}$, and $y \neq u_{k+1}$ in $u_{k+1}^{*}$. By Corollary 2.4, we may assume that $W=\{x, y\}$.

Suppose that $u_{k}^{*}$ is type $(N)$. Then $x \not u_{k}$, implying $\operatorname{dist}\left(x, u_{k}\right)=\operatorname{dist}\left(x, u_{k+2}\right)=2$ and $\operatorname{dist}\left(y, u_{k}\right)=\operatorname{dist}\left(y, u_{k+2}\right)=1$. Thus neither $x$ nor $y$ resolves $u_{k}, u_{k+2}$.

Suppose that $u_{k+1}^{*}$ is type $(N)$. Then $y \nsim u_{k+1}$, implying $\operatorname{dist}\left(x, u_{k-1}\right)=\operatorname{dist}\left(x, u_{k+1}\right)=$ 1 and $\operatorname{dist}\left(y, u_{k-1}\right)=\operatorname{dist}\left(y, u_{k+1}\right)=2$. Thus neither $x$ nor $y$ resolves $u_{k-1}, u_{k+1}$.

Suppose that $u_{k}^{*}$ and $u_{k+1}^{*}$ are both type $(K)$. Then $x \sim u_{k}$ and $y \sim u_{k+1}$, implying $\operatorname{dist}\left(x, u_{k}\right)=\operatorname{dist}\left(x, u_{k+1}\right)=1$ and $\operatorname{dist}\left(y, u_{k}\right)=\operatorname{dist}\left(y, u_{k+1}\right)=1$. Thus neither $x$ nor $y$ resolves $u_{k}, u_{k+1}$.

In each case we have a contradiction.

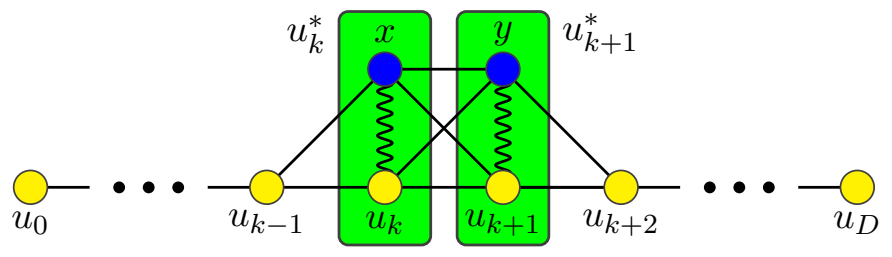

Figure 17: In Case 1(b)(i).

Case 1(b)(ii). $\alpha\left(G^{*}\right)=2, u_{D-1}^{*}$ is not type (1), and $u_{D}^{*}$ is not type (1): As illustrated in Figure 18, consider $x \neq u_{D-1}$ in $u_{D-1}^{*}$ and $y \neq u_{D}$ in $u_{D}^{*}$. By Corollary 2.4, we may assume that $W=\{x, y\}$.

First suppose that $u_{D}^{*}$ is of type $(N)$. Then $y \neq u_{D}$, implying $\operatorname{dist}\left(x, u_{D-2}\right)=$ $\operatorname{dist}\left(x, u_{D}\right)=1$ and $\operatorname{dist}\left(y, u_{D-2}\right)=\operatorname{dist}\left(y, u_{D}\right)=2$. Thus neither $x$ nor $y$ resolves $u_{D-2}, u_{D}$, which is a contradiction.

Suppose that $u_{D}^{*}$ and $u_{D-1}^{*}$ are both type $(K)$. Then $x \sim u_{D-1}$ and $y \sim u_{D}$, implying $\operatorname{dist}\left(x, u_{D-1}\right)=\operatorname{dist}\left(x, u_{D}\right)=1$ and $\operatorname{dist}\left(y, u_{D-1}\right)=\operatorname{dist}\left(y, u_{D}\right)=1$. Thus neither $x$ nor $y$ resolves $u_{D-1}, u_{D}$, which is a contradiction.

Case 1(c). $\alpha\left(G^{*}\right)=2$ and $u_{k-1}^{*}$ is type $(N)$, and $u_{k+1}^{*}$ is type $(N)$ for some $k \in$ $[2, D-1]$ : As illustrated in Figure 19 , consider $x \neq u_{k-1}$ in $u_{k-1}^{*}$ and $y \neq u_{k+1}$ in $u_{k+1}^{*}$. By Corollary 2.4. we may assume that $W=\{x, y\}$. Since $x \not u_{k-1}$ and $y \not u_{k+1}$, we have $\operatorname{dist}\left(x, u_{k-1}\right)=\operatorname{dist}\left(x, u_{k+1}\right)=2$ and $\operatorname{dist}\left(y, u_{k-1}\right)=\operatorname{dist}\left(y, u_{k+1}\right)=2$. Thus neither $x$ nor $y$ resolves $u_{k-1}, u_{k+1}$, which is a contradiction. 


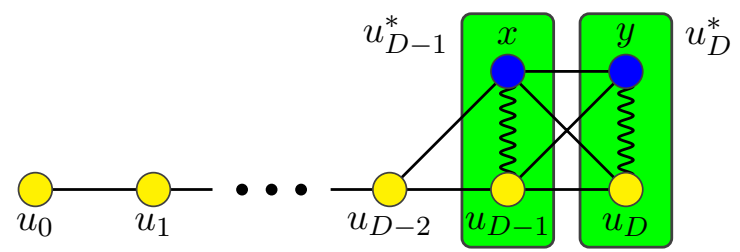

Figure 18: In Case 1(b)(ii).

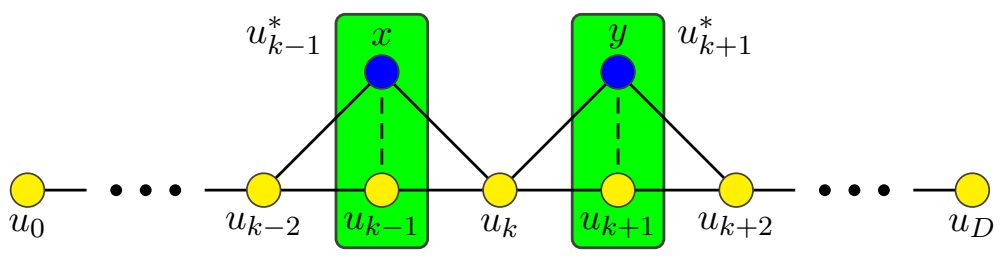

Figure 19: $\{x, y\}$ does not resolve $u_{k-1}, u_{k+1}$ in Case 1(c).

Case 1(d). $\alpha\left(G^{*}\right)=3, u_{k-1}^{*}$ is type $(N), u_{k}^{*}$ is type $(K)$ or $(N)$, and $u_{k+1}^{*}$ is type $(N)$ for some $k \in[2, D-1]$ : As illustrated in Figure 20, consider $x \neq u_{k-1}$ in $u_{k-1}^{*}, y \neq u_{k}$ in $u_{k}^{*}$, and $z \neq u_{k+1}$ in $u_{k+1}^{*}$. By Corollary 2.4 we may assume that $W=\{x, y, z\}$. Now $x \nsim u_{k-1}$ and $z \nsim u_{k+1}$. Thus $\operatorname{dist}\left(x, u_{k-1}\right)=\operatorname{dist}\left(x, u_{k+1}\right)=2, \operatorname{dist}\left(y, u_{k-1}\right)=\operatorname{dist}\left(y, u_{k+1}\right)=1$, and $\operatorname{dist}\left(z, u_{k-1}\right)=\operatorname{dist}\left(z, u_{k+1}\right)=2$. Thus $\{x, y, z\}$ does not resolve $u_{k-1}, u_{k+1}$, which is a contradiction.

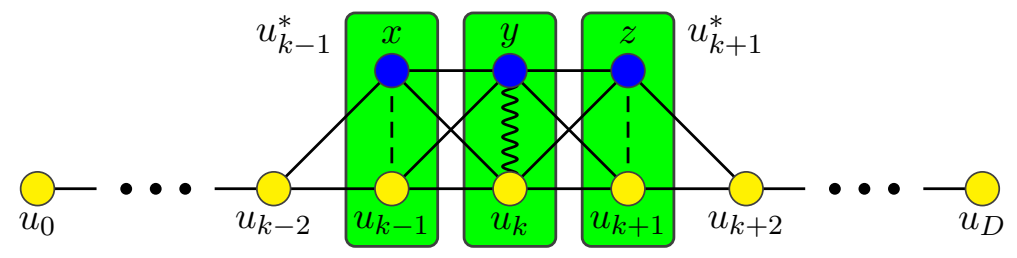

Figure 20: $\{x, y, z\}$ does not resolve $u_{k-1}, u_{k+1}$ in Case 1(d).

Case 2. $G^{*} \cong P_{D+1, k}$ for some $k \in[3, D-1]$ : Thus $G^{*}$ is path $\left(u_{0}^{*}, u_{1}^{*}, \ldots, u_{D}^{*}\right)$ plus one vertex $w^{*}$ adjacent to $u_{k-1}^{*}$. As illustrated in Figure 21, suppose that every vertex of $G^{*}$ is of type (1), except for $u_{k-2}^{*}, u_{k}^{*}$ and $w^{*}$ which are type $(1 N)$, and $u_{k-1}^{*}$ which is of any type. In this case $n-D-1=\alpha\left(G^{*}\right)+1$. Consequently, it suffices to prove that $\alpha\left(G^{*}\right)+1$ vertices do not resolve $G$. Suppose there is a resolving set $W$ in $G$ of cardinality $\alpha\left(G^{*}\right)+1$. By Corollary 2.4, we can assume that $W$ contains the $\alpha\left(G^{*}\right)$ twins of $u_{k-2}, u_{k-1}, u_{k}$ and $w$ (if they exist), and another vertex of $G$. Let $x_{k-2}, x_{k-1}, x_{k}$ and $y$ respectively be twin vertices of $u_{k-2}, u_{k-1}, u_{k}$ and $w$ (if they exist). Then $x_{k-2} \nsim u_{k-2}, x_{k} \nsim u_{k}$, and $y \nsim w$. Thus the distance from $x_{k-2}$ (respectively $x_{k-1}, x_{k}, y$ ) to any vertex of $u_{k-2}, u_{k}, w$ is 2 (respectively $1,2,2)$. Hence any set of twins of vertices in $\left\{u_{k-2}, u_{k-1}, u_{k}, w\right\}$ (if they 
exist) does not resolve $\left\{u_{k-2}, u_{k}, w\right\}$. Moreover, if $i \in[0, k-1]$ then $u_{i}$ does not resolve $u_{k}, w$; if $i \in[k-1, D]$ then $u_{i}$ does not resolve $u_{k-2}, w$; and $w$ does not resolve $u_{k-2}, u_{k}$. Therefore, $\alpha\left(G^{*}\right)+1$ vertices do not resolve $G$.

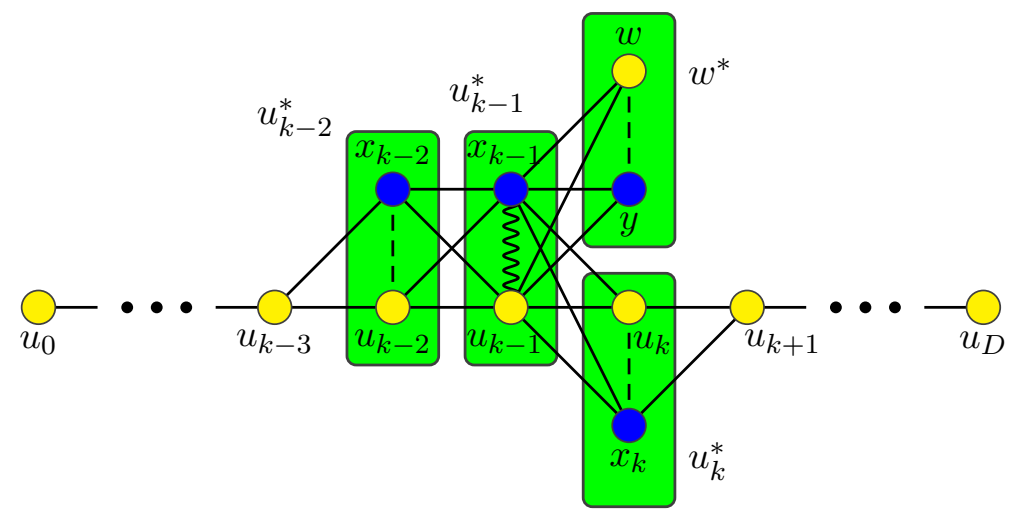

Figure 21: $\left\{x_{k-2}, x_{k-1}, x_{k}, y\right\}$ does not resolve $\left\{u_{k-2}, u_{k}, w\right\}$ in Case 2 .

Case 3. $G^{*} \cong P_{D+1, k}^{\prime}$ for some $k \in[2, D-1]$ : Thus $G^{*}$ is path $\left(u_{0}^{*}, u_{1}^{*}, \ldots, u_{D}^{*}\right)$ plus one vertex $w^{*}$ adjacent to $u_{k-1}^{*}$ and $u_{k}^{*}$. As illustrated in Figure 22, suppose that every vertex of $G^{*}$ is type (1) except for $u_{k-1}^{*}, u_{k}^{*}$, and $w^{*}$ which are of type $(1 K)$. In this case, $n-D-1=\alpha\left(G^{*}\right)+1$. Consequently, it suffices to prove that $\alpha\left(G^{*}\right)+1$ vertices do not resolve $G$. Suppose there is a resolving set $W$ in $G$ of cardinality $\alpha\left(G^{*}\right)+1$. By Corollary 2.4, we may assume that $W$ contains exactly the $\alpha\left(G^{*}\right)$ twin vertices of $u_{k-1}, u_{k}$ and $w$ (if they exist), and another vertex of $G$. Let $x_{k-1}, x_{k}$, and $y$ respectively be twins of $u_{k-1}, u_{k}$ and $w$ (if they exist). Hence $x_{k-1} \sim u_{k-1}, x_{k} \sim u_{k}$ and $y \sim w$. Consequently, $u_{k-1}, u_{k}$ and $w$ are at distance 1 from $x_{k-1}, x_{k}$ and $y$. Thus any set of twins of vertices in $\left\{u_{k-1}, u_{k}, w\right\}$ (if they exist) does not resolve $\left\{u_{k-1}, u_{k}, w\right\}$. Moreover, if $i \in[0, k-1]$ then $u_{i}$ does not resolve $u_{k}, w$; if $i \in[k, D]$ then $u_{i}$ does not resolve $u_{k-1}, w$; and $w$ does not resolve $u_{k-1}, u_{k}$. Thus $\alpha\left(G^{*}\right)+1$ vertices do not resolve $G$.

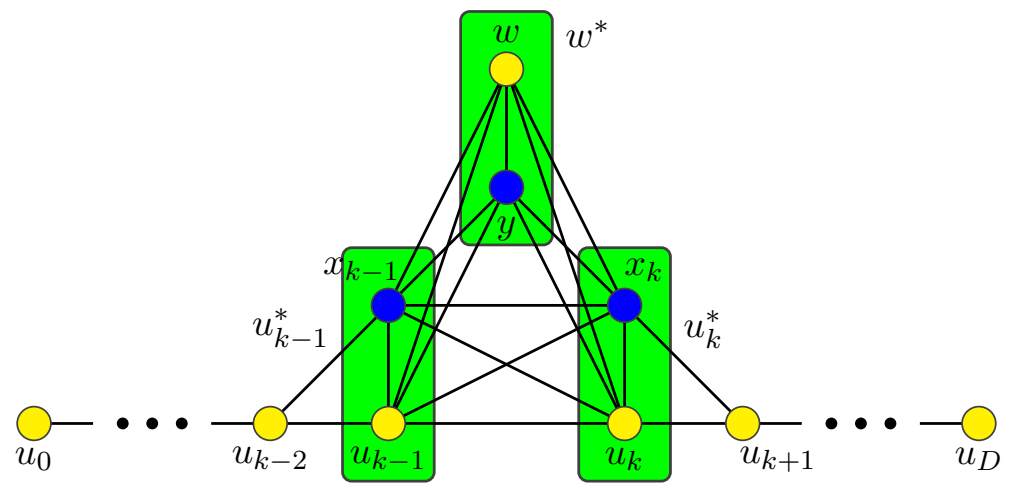

Figure 22: $\left\{x_{k-1}, x_{k}, y\right\}$ does not resolve $\left\{u_{k-1}, u_{k}, w\right\}$ in Case 3 . 


\section{Graphs with Maximum Order}

In this section we determine the maximum order of a graph in $\mathcal{G}_{\beta, D}$.

Theorem 3.1. For all integers $D \geqslant 2$ and $\beta \geqslant 1$, the maximum order of a connected graph with diameter $D$ and metric dimension $\beta$ is

$$
\left(\left\lfloor\frac{2 D}{3}\right\rfloor+1\right)^{\beta}+\beta \sum_{i=1}^{\lceil D / 3\rceil}(2 i-1)^{\beta-1} .
$$

First we prove the upper bound in Theorem 3.1 .

Lemma 3.2. For every graph $G \in \mathcal{G}_{\beta, D}$,

$$
|V(G)| \leqslant\left(\left\lfloor\frac{2 D}{3}\right\rfloor+1\right)^{\beta}+\beta \sum_{i=1}^{\lceil D / 3\rceil}(2 i-1)^{\beta-1} .
$$

Proof. Let $S$ be a metric basis of $G$. Let $k \in[0, D]$ be specified later. For each vertex $v \in S$ and integer $i \in[0, k]$, let $N_{i}(v):=\{x \in V(G): \operatorname{dist}(v, x)=i\}$.

Consider two vertices $x, y \in N_{i}(v)$. There is a path from $x$ to $v$ of length $i$, and there is a path from $y$ to $v$ of length $i$. Thus $\operatorname{dist}(x, y) \leqslant 2 i$. Hence for each vertex $u \in S$, the difference between $\operatorname{dist}(u, x)$ and $\operatorname{dist}(u, y)$ is at most $2 i$. Thus the distance vector of $x$ with respect to $S$ has an $i$ in the coordinate corresponding to $v$, and in each other coordinate, there are at most $2 i+1$ possible values. Therefore $\left|N_{i}(v)\right| \leqslant(2 i+1)^{\beta-1}$.

Consider a vertex $x \in V(G)$ that is not in $N_{i}(v)$ for all $v \in S$ and $i \in[0, k]$. Then $\operatorname{dist}(x, v) \geqslant k+1$ for all $v \in S$. Thus the distance vector of $x$ with respect to $S$ consists of $\beta$ numbers in $[k+1, D]$. Thus there are at most $(D-k)^{\beta}$ such vertices. Hence

$$
\begin{aligned}
|V(G)| & \leqslant(D-k)^{\beta}+\sum_{v \in S} \sum_{i=0}^{k}\left|N_{i}(v)\right| \\
& \leqslant(D-k)^{\beta}+\beta \sum_{i=0}^{k}(2 i+1)^{\beta-1} .
\end{aligned}
$$

Note that with $k=0$ we obtain the bound $|V(G)| \leqslant D^{\beta}+\beta$, independently due to Khuller et al. 21] and Chartrand et al. [5]. Instead we define $k:=\lceil D / 3\rceil-1$. Then $k \in[0, D]$ and

$$
\begin{aligned}
|V(G)| & \leqslant\left(D-\left\lceil\frac{D}{3}\right\rceil+1\right)^{\beta}+\beta \sum_{i=0}^{\lceil D / 3\rceil-1}(2 i+1)^{\beta-1} \\
& =\left(\left\lfloor\frac{2 D}{3}\right\rfloor+1\right)^{\beta}+\beta \sum_{i=1}^{\lceil D / 3\rceil}(2 i-1)^{\beta-1} .
\end{aligned}
$$


To prove the lower bound in Theorem 3.1 we construct a graph $G \in \mathcal{G}_{\beta, D}$ with as many vertices as in Equation (2). The following definitions apply for the remainder of this section. Let $A:=\lceil D / 3\rceil$ and $B:=\lceil D / 3\rceil+\lfloor D / 3\rfloor$. Consider the following subsets of $\mathbb{Z}^{\beta}$. Let

$$
Q:=\left\{\left(x_{1}, \ldots, x_{\beta}\right): A \leqslant x_{i} \leqslant D, i \in[1, \beta]\right\} .
$$

For each $i \in[1, \beta]$ and $r \in[0, A-1]$, let

$$
P_{i, r}:=\left\{\left(x_{1}, \ldots, x_{i-1}, r, x_{i+1}, \ldots, x_{\beta}\right): x_{j} \in[B-r, B+r], j \neq i\right\} .
$$

Let $P_{i}:=\bigcup\left\{P_{i, r}: r \in[0, A-1]\right\}$ and $P:=\bigcup\left\{P_{i}: i \in[1, \beta]\right\}$. Let $G$ be the graph with vertex set $V(G):=Q \cup P$, where two vertices $\left(x_{1}, \ldots, x_{\beta}\right)$ and $\left(y_{1}, \ldots, y_{\beta}\right)$ in $V(G)$ are adjacent if and only if $\left|y_{i}-x_{i}\right| \leqslant 1$ for each $i \in[1, \beta]$. Figures 23 and 24 illustrate $G$ for $\beta=2$ and $\beta=3$ respectively.

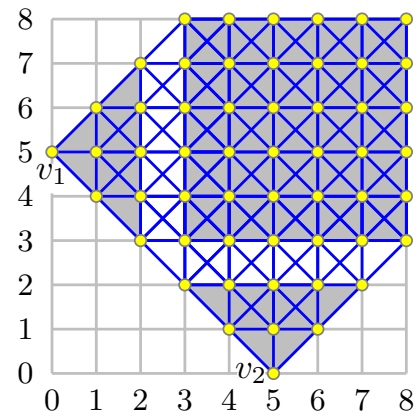

(a) $D=8 \equiv 2(\bmod 3)$

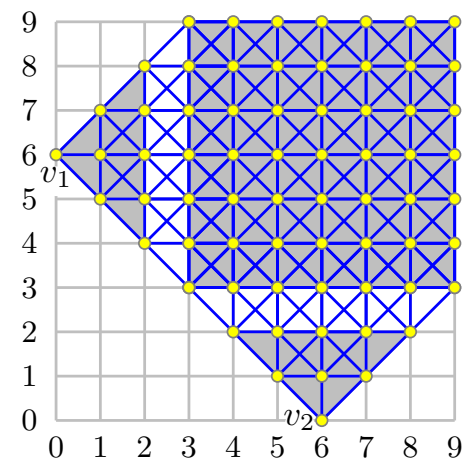

(b) $D=9 \equiv 0(\bmod 3)$

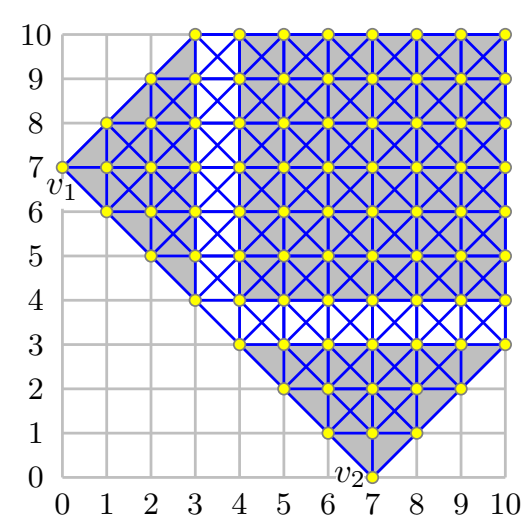

(c) $D=10 \equiv 1(\bmod 3)$

Figure 23: The graph $G$ with $\beta=2$. The shaded regions are $Q, P_{1}$, and $P_{2}$.

Lemma 3.3. For all positive integers $D$ and $\beta$,

$$
|V(G)|=\left(\left\lfloor\frac{2 D}{3}\right\rfloor+1\right)^{\beta}+\beta \sum_{i=1}^{\lceil D / 3\rceil}(2 i-1)^{\beta-1} .
$$

Proof. Observe that each coordinate of each vertex in $Q$ is at least $A$, and each vertex in $P$ has some coordinate less than $A$. Thus $Q \cap P=\emptyset$. Each vertex in $P_{j}(j \neq i)$ has an $i$-coordinate at least $B-r \geqslant B-(A-1)=\lfloor D / 3\rfloor+1$, and each vertex in $P_{i}$ has an $i$-coordinate of $r \leqslant A-1<\lfloor D / 3\rfloor+1$. Thus $P_{i} \cap P_{j}=\emptyset$ whenever $i \neq j$. Each vertex in 


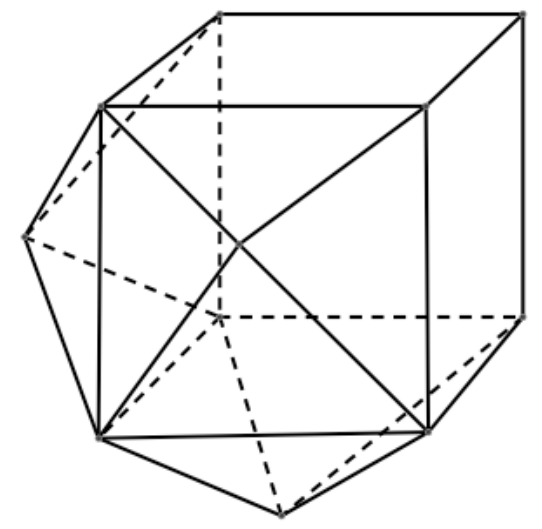

Figure 24: The convex hull of $V(G)$ with $\beta=3$.

$P_{i, r}$ has an $i$-coordinate of $r$. Thus $P_{i, r} \cap P_{i, s}=\emptyset$ whenever $r \neq s$. Thus

$$
\begin{aligned}
|V(G)| & =|Q|+\sum_{i=1}^{\beta} \sum_{r=0}^{A-1}\left|P_{i, r}\right| \\
& =\left(D-\left(\left\lceil\frac{D}{3}\right\rceil-1\right)\right)^{\beta}+\beta \sum_{r=0}^{A-1}(2 r+1)^{\beta-1} \\
& =\left(\left\lfloor\frac{2 D}{3}\right\rfloor+1\right)^{\beta}+\beta \sum_{r=1}^{A}(2 r-1)^{\beta-1} .
\end{aligned}
$$

We now determine the diameter of $G$. For distinct vertices $x=\left(x_{1}, \ldots, x_{\beta}\right)$ and $y=\left(y_{1}, \ldots, y_{\beta}\right)$ of $G$, let $z(x, y):=\left(z_{1}, \ldots, z_{\beta}\right)$ where

$$
z_{i}= \begin{cases}x_{i} & \text { if } x_{i}=y_{i} \\ x_{i}+1 & \text { if } x_{i}<y_{i} \\ x_{i}-1 & \text { if } x_{i}>y_{i}\end{cases}
$$

Lemma 3.4. $z(x, y) \in V(G)$ for all distinct vertices $x, y \in V(G)$.

Proof. The following observations are an immediate consequence of the definition of $z(x, y)$, where $h, k \in \mathbb{Z}$ and $j \in[1, \beta]$ :

(i) if $x_{j}, y_{j} \in[h, k]$ then $z_{j} \in[h, k]$;

(ii) if $x_{j} \in[h, k]$ then $z_{j} \in[h-1, k+1]$; and

(iii) if $x_{j} \in[h, k]$ and $y_{j} \in\left[h^{\prime}, k^{\prime}\right]$ for some $h^{\prime}>h$ and $k^{\prime}<k$, then $z_{j} \in[h+1, k-1]$. 
We distinguish the following cases:

(1) $x, y \in Q$ : Then $x_{j}, y_{j} \in[A, D]$ for all $j$. Thus $z_{j} \in[A, D]$ by (i). Hence $z \in Q$.

(2) $x \in P$ and $y \in Q$ : Without loss of generality, $x \in P_{1, r}$; that is, $x=\left(r, x_{2}, \ldots, x_{\beta}\right)$, where $r \in[0, A-1]$ and $x_{j} \in[B-r, B+r]$. Since $y \in Q$, we have $y_{1} \geqslant A>r$. Thus $z=\left(r+1, z_{2}, \ldots, z_{\beta}\right)$.

(2.1) $z_{1}=r+1<A$ : By (ii), $z_{j} \in[B-r-1, B+r+1]$ for every $j \neq 1$. Thus $z \in P_{1, r+1}$.

(2.2) $z_{1}=r+1=A$ : Then $z_{1} \in[A, D]$. On the other hand, if $j \neq 2$ then $y_{j} \geqslant A$, and since $x \in P_{1, r}$ and $r=A-1$, we have $x_{j} \geqslant B-r=\lfloor D / 3\rfloor+1 \geqslant\lceil D / 3\rceil=A$. Thus $z_{j} \geqslant A$. Hence $z \in Q$.

(3) $x \in Q$ and $y \in P$ : Without loss of generality, $y \in P_{1, r}$. That is, $y=\left(r, y_{2}, \ldots, y_{\beta}\right)$, where $r \in[0, A-1], y_{j} \in[B-r, B+r]$, and $x=\left(x_{1}, \ldots, x_{\beta}\right)$, where $x_{j} \geqslant A$ for all $j$. That is, $x_{1}>r=y_{1}$, and therefore $z=\left(x_{1}-1, z_{2}, \ldots, z_{\beta}\right)$.

(3.1) $z_{1}=x_{1}-1 \geqslant A$ : Since $x_{j}, y_{j} \geqslant A$, by (i), $z_{j} \geqslant A$ for every $j \neq 1$. That is, $z \in Q$.

(3.2) $z_{1}=x_{1}-1=A-1$ : Since $r \leqslant A-1$, we have $y_{j} \in[B-r, B+r] \subseteq$ $[B-(A-1), B+A-1]$ for all $j \notin\{1,2\}$. Now $B-A=\lfloor D / 3\rfloor \leqslant\lceil D / 3\rceil=A$ and $D \leqslant\lfloor D / 3\rfloor+2\lceil D / 3\rceil=A+B$. Thus $x_{j} \in[B-A, B+A]$. By (iii), $z_{j} \in[B-(A-1), B+A-1]$. That is, $z \in P_{1, A-1}$.

(4) $x, y \in P_{h}$ : Without loss of generality, $x, y \in P_{1}$. Thus $x=\left(r, x_{2}, \ldots, x_{\beta}\right)$ for some $r \in[0, A-1]$ with $x_{j} \in[B-r, B+r]$ for all $j \neq 1$, and $y=\left(s, y_{2}, \ldots, y_{\beta}\right)$ for some $s \in[0, A-1]$ with $y_{j} \in[B-s, B+s]$ for all $j \neq 1$.

(4.1) $r=s$ : Then $z=\left(r, z_{2}, \ldots, z_{\beta}\right)$. By (i), $z_{j} \in[B-r, B+r]$ for all $j \neq 1$. Thus $z \in P_{1, r}$.

(4.2) $r<s$ : Then $z=\left(r+1, z_{2}, \ldots, z_{\beta}\right)$. By (ii), $z_{j} \in[B-(r+1), B+r+1]$ for all $j \neq 1$. Thus $z \in P_{1, r+1}$.

(4.3) $r>s$ : Then $z=\left(r-1, z_{2}, \ldots, z_{\beta}\right)$. By (iii), $z_{j} \in[B-(r-1), B+r-1]$. Thus $z \in P_{1, r-1}$.

(5) $x \in P_{h}, y \in P_{k}$ and $h \neq k$ : Without loss of generality, $x \in P_{1}$ and $y \in P_{2}$. Thus $x=\left(r, x_{2}, \ldots, x_{\beta}\right)$ for some $r \in[0, A-1]$ with $x_{j} \in[B-r, B+r]$ for all $j \neq 1$, and $y=\left(y_{1}, s, y_{3}, \ldots, y_{\beta}\right)$ for some $s \in[0, A-1]$ with $y_{j} \in[B-s, B+s]$ for all $j \neq 2$. Hence $r<A \leqslant y_{1}$ and $s<A \leqslant x_{2}$, implying $z=\left(r+1, x_{2}-1, z_{3}, \ldots, z_{\beta}\right)$.

(5.1) $z_{1}=r+1<A$ : Now $x_{j} \in[B-r, B+r]$ for $j \neq 1$. Thus $z_{j} \in[B-r-1, B+r+1]$ by (ii). Thus $z \in P_{1, r+1}$.

(5.2) $z_{1}=r+1=A$ : Consider the following subcases: 
(5.2.1) $z_{2}=x_{2}-1 \geqslant A$ : By hypotheses, $z_{1}, z_{2} \geqslant A$. For $j \notin\{1,2\}$, since $x_{j}, y_{j} \geqslant A$, (i) implies that $z_{j} \geqslant A$. Thus $z \in Q$.

(5.2.2) $z_{2}=x_{2}-1=A-1$ : In this case $x=\left(A-1, A, x_{3}, \ldots, x_{\beta}\right), z=(A, A-$ $\left.1, z_{3}, \ldots, z_{\beta}\right)$, and $s \leqslant A-1=r$. Since $x \in P_{1, A-1}$, we have $z_{1}=x_{2}=A \in$ $[B-(A-1), B+A-1]$. For $j \notin\{1,2\}$, since $x_{j} \in[B-(A-1), B+A-1]$ and $y_{j} \in[B-s, B+s]$, where $s \leqslant r=A-1$, (i) implies that $z_{j} \in$ $[B-(A-1), B+A-1]$. That is, $z \in P_{2, A-1}$.

Lemma 3.5. For all vertices $x=\left(x_{1}, \ldots, x_{\beta}\right)$ and $y=\left(y_{1}, \ldots, y_{\beta}\right)$ of $G$,

$$
\operatorname{dist}(x, y)=\max \left\{\left|y_{i}-x_{i}\right|: i \in[1, \beta]\right\} \leqslant D .
$$

Proof. For each $i \in[1, \beta]$, dist $(x, y) \geqslant\left|x_{i}-y_{i}\right|$ since on every $x y$-path $P$, the $i$-coordinates of each pair of adjacent vertices in $P$ differ by at most 1 . This proves the lower bound $\operatorname{dist}(x, y) \geqslant \max _{i}\left|y_{i}-x_{i}\right|$.

Now we prove the upper bound $\operatorname{dist}(x, y) \leqslant \max _{i}\left|y_{i}-x_{i}\right|$ by induction. If $\max _{i} \mid y_{i}-$ $x_{i} \mid=1$ then $x$ and $y$ are adjacent, and thus $\operatorname{dist}(x, y)=1$. Otherwise, let $z:=z(x, y)$. By the definition of $z(x, y)$, for all $i \in[1, \beta]$ we have $\left|y_{i}-z_{i}\right|=\left|y_{i}-x_{i}\right|-1$ unless $x_{i}=y_{i}$. Thus $\max _{i}\left|y_{i}-z_{i}\right|=\max _{i}\left|y_{i}-x_{i}\right|-1$. By induction, $\operatorname{dist}(z, y) \leqslant \max _{i}\left|y_{i}-z_{i}\right|=\max _{i}\left|y_{i}-x_{i}\right|-1$. By Lemma 3.4, $z$ is a vertex of $G$, and by construction, $x$ and $z$ are adjacent. Thus $\operatorname{dist}(x, y) \leqslant \operatorname{dist}(z, y)+1=\max _{i}\left|y_{i}-x_{i}\right|$, as desired.

Lemma 3.5 implies that $G$ has diameter $D$. Let $S:=\left\{v_{1}, \ldots, v_{\beta}\right\}$, where

$$
v_{i}=(\underbrace{B, \ldots, B}_{i-1}, 0, B, \ldots, B) .
$$

Observe that each $v_{i} \in P_{i}$. We now prove that $S$ is a metric basis of $G$.

Lemma 3.6. $\operatorname{dist}\left(x, v_{i}\right)=x_{i}$ for every vertex $x=\left(x_{1}, \ldots, x_{\beta}\right)$ of $G$ and for each $v_{i} \in S$.

Proof. Let $v_{i, j}$ be the $j$-th coordinate of $v_{i}$; that is, $v_{i, i}=0$ and $v_{i, j}=B$ for $i \neq j$. Then $\operatorname{dist}\left(x, v_{i}\right)=\max \left\{\left|v_{i, j}-x_{j}\right|: 1 \leqslant j \leqslant \beta\right\}=\max \left\{x_{i}, \max \left\{\left|B-x_{j}\right|: 1 \leqslant j \leqslant \beta, j \neq i\right\}\right\}$. We claim that $\left|B-x_{j}\right| \leqslant x_{i}$ for each $j \neq i$, implying $\operatorname{dist}\left(x, v_{i}\right)=x_{i}$, as desired.

First suppose that $x \in Q$. Then $A \leqslant x_{j} \leqslant D$. Thus $\left|B-x_{j}\right| \leqslant$ $\max \{B-A, D-B\}=\max \{\lfloor D / 3\rfloor, D-B\} \leqslant \max \{\lfloor D / 3\rfloor,\lceil D / 3\rceil\}=\lceil D / 3\rceil \leqslant x_{i}$.

Now suppose that $x \in P_{k, r}$ for some $k \neq i$ and for some $r$. Then $x_{i} \geqslant B-r \geqslant$ $B-(\lceil D / 3\rceil-1)=\lfloor D / 3\rfloor+1 \geqslant\lceil D / 3\rceil$. Now $\left|B-x_{j}\right| \leqslant r \leqslant\lceil D / 3\rceil-1$. Thus $\left|B-x_{j}\right| \leqslant x_{i}$.

Finally suppose that $x \in P_{i, r}$ for some $r$. Then $\left|B-x_{j}\right| \leqslant r=x_{i}$.

Lemma 3.6 implies that the metric coordinates of a vertex $x \in V(G)$ with respect to $S$ are its coordinates as elements of $\mathbb{Z}^{\beta}$. Therefore $S$ resolves $G$. Thus $G$ has metric dimension at most $|S|=\beta$. 
If the metric dimension of $G$ was less than $\beta$, then by Lemma 3.2,

$$
\left(\left\lfloor\frac{2 D}{3}\right\rfloor+1\right)^{\beta}+\beta \sum_{i=1}^{\lceil D / 3\rceil}(2 i-1)^{\beta-1}=|V(G)| \leqslant\left(\left\lfloor\frac{2 D}{3}\right\rfloor+1\right)^{\beta-1}+(\beta-1) \sum_{i=1}^{\lceil D / 3\rceil}(2 i-1)^{\beta-2},
$$

which is a contradiction. Thus $G$ has metric dimension $\beta$, and $G \in \mathcal{G}_{\beta, D}$. This completes the proof of Theorem 3.1 .

\section{References}

[1] Zuzana Beerliova, Felix Eberhard, Thomas Erlebach, Alexander Hall, Michael Hoffmann, Matúš Mihal'ák, and L. Shankar Ram. Network discovery and verification. IEEE J. on Selected Areas in Communications, 24(12):2168-2181, 2006.

[2] Robert C. Brigham, Gary Chartrand, Ronald D. Dutton, and Ping Zhang. Resolving domination in graphs. Math. Bohem., 128(1):25-36, 2003.

[3] José Cáceres, Carmen Hernando, Mercè Mora, ignacio M. Pelayo, María L. Puertas, Carlos Seara, and David R. Wood. On the metric dimension of cartesian products of graphs. SIAM J. Discrete Math., 21(2):423-441, 2007. http://dx.doi.org/10.1137/050641867.

[4] Glenn G. Chappell, John Gimbel, and Chris Hartman. Bounds on the metric and partition dimensions of a graph. Ars Combin., 88:349-366, 2008.

[5] Gary Chartrand, Linda Eroh, Mark A. Johnson, and Ortrud R. Oellermann. Resolvability in graphs and the metric dimension of a graph. Discrete Appl. Math., 105(1-3):99-113, 2000. http://dx.doi.org/10.1016/ S0166-218X (00) 00198-0

[6] Gary Chartrand, Christopher Poisson, and Ping Zhang. Resolvability and the upper dimension of graphs. Comput. Math. Appl., 39(12):19-28, 2000.

[7] Gary Chartrand and Ping Zhang. The theory and applications of resolvability in graphs. A survey. In Proc. 34th Southeastern International Conf. on Combinatorics, Graph Theory and Computing, vol. 160 of Congr. Numer., pp. 47-68. 2003.

[8] Vašek Chvátal. Mastermind. Combinatorica, 3(3-4):325-329, 1983.

[9] James Currie and Ortrud R. Oellermann. The metric dimension and metric independence of a graph. J. Combin. Math. Combin. Comput., 39:157-167, 2001.

[10] Reinhard Diestel. Graph theory, vol. 173 of Graduate Texts in Mathematics. Springer, 2nd edn., 2000. http://diestel-graph-theory.com/index.html.

[11] Paul ERdős And Alfréd RÉNYI. On two problems of information theory. Magyar Tud. Akad. Mat. Kutató Int. Közl., 8:229-243, 1963.

[12] Wayne Goddard. Static mastermind. J. Combin. Math. Combin. Comput., 47:225236, 2003. 
[13] Wayne Goddard. Mastermind revisited. J. Combin. Math. Combin. Comput., 51:215-220, 2004.

[14] Don L. Greenwell. Mastermind. J. Recr. Math., 30:191-192, 1999-2000.

[15] Richard K. Guy and Richard J. Nowakowski. Coin-weighing problems. Amer. Math. Monthly, 102(2):164, 1995. http://www. jstor.org/stable/2975353.

[16] Frank Harary and Robert A. Melter. On the metric dimension of a graph. Ars Combinatoria, 2:191-195, 1976.

[17] Carmen Hernando, Mercé Mora, Peter J. Slater, and David R. Wood. Fault-tolerant metric dimension of graphs. In Convexity in discrete structures, vol. 5 of Ramanujan Math. Soc. Lect. Notes Ser., pp. 81-85. Ramanujan Math. Soc., 2008.

[18] H. Iswadi, E. T. Baskoro, R. Simanjuntak, and A. N. M. Salman. The metric dimension of graph with pendant edges. J. Combin. Math. Combin. Comput., 65:139-145, 2008.

[19] Imran Javaid, M. Tariq Rahim, and Kashif Ali. Families of regular graphs with constant metric dimension. Util. Math., 75:21-33, 2008.

[20] Grigori Kabatianski, V. S. Lebedev, and J. Thorpe. The Mastermind game and the rigidity of Hamming spaces. In Proc. IEEE International Symposium on Information Theory (ISIT '00), p. 375. IEEE, 2000.

[21] Samir Khuller, Balaji Raghavachari, and Azriel Rosenfeld. Landmarks in graphs. Discrete Appl. Math., 70(3):217-229, 1996. http://dx.doi.org/10. 1016/0166-218X (95) 00106-2.

[22] Bernt Lindström. On a combinatory detection problem. I. Magyar Tud. Akad. Mat. Kutató Int. Közl., 9:195-207, 1964.

[23] Paul Manuel, Bharati Rajan, Indra Rajasingh, and M. Chris Monica. Landmarks in torus networks. J. Discrete Math. Sci. Cryptogr., 9(2):263-271, 2006.

[24] Paul Manuel, Bharati Rajan, Indra Rajasingh, and Chris Monica M. On minimum metric dimension of honeycomb networks. J. Discrete Algorithms, 6(1):20-27, 2008. http://dx.doi.org/10.1016/j.jda.2006.09.002.

[25] Ortrud R. Oellermann and Joel Peters-Fransen. The strong metric dimension of graphs and digraphs. Discrete Appl. Math., 155(3):356-364, 2007. http://dx.doi.org/10.1016/j.dam.2006.06.009.

[26] Joel Peters-Fransen and Ortrud R. Oellermann. The metric dimension of the cartesian product of graphs. Utilitas Math., 69:33-41, 2006.

[27] Christopher Poisson and Ping Zhang. The metric dimension of unicyclic graphs. J. Combin. Math. Combin. Comput., 40:17-32, 2002.

[28] Bharati Rajan, Indra Rajasingh, M. Chris Monica, and Paul Manuel. Metric dimension of enhanced hypercube networks. J. Combin. Math. Combin. Comput., 67:5-15, 2008.

[29] Varaporn Saenpholphat and Ping Zhang. Connected resolvability of graphs. Czechoslovak Math. J., 53(128)(4):827-840, 2003. 
[30] Varaporn Saenpholphat and Ping Zhang. Conditional resolvability in graphs: a survey. Int. J. Math. Math. Sci., (37-40):1997-2017, 2004.

[31] Varaporn Saenpholphat and Ping Zhang. Detour resolvability of graphs. In Proc. of 35th Southeastern International Conf. on Combinatorics, Graph Theory and Computing, vol. 169, pp. 3-21. 2004.

[32] Varaporn Saenpholphat and Ping Zhang. On connected resolving decompositions in graphs. Czechoslovak Math. J., 54(129)(3):681-696, 2004.

[33] S. W. Saputro, Edy Tri Baskoro, A. N. M. Salman, and D. Suprijanto. The metric dimensions of a complete $n$-partite graph and its Cartesian product with a path. J. Combin. Math. Combin. Comput., 71:283-293, 2009.

[34] András SEbő And ERIC TANnier. On metric generators of graphs. Math. Oper. Res., 29(2):383-393, 2004.

[35] B. Shanmukha, B. Sooryanarayana, and K. S. Harinath. Metric dimension of wheels. Far East J. Appl. Math., 8(3):217-229, 2002.

[36] Peter J. Slater. Leaves of trees. In Proc. 6th Southeastern Conf. on Combinatorics, Graph Theory, and Computing, vol. 14 of Congr. Numer., pp. 549-559. Utilitas Math., 1975.

[37] Peter J. Slater. Dominating and reference sets in a graph. J. Math. Phys. Sci., 22(4):445-455, 1988.

[38] Staffan Söderberg and Harold S. Shapiro. A combinatory detection problem. Amer. Math. Monthly, 70:1066, 1963. http://www.jstor.org/stable/ 2312835 .

[39] B. Sooryanarayana. On the metric dimension of a graph. Indian J. Pure Appl. Math., 29(4):413-415, 1998.

[40] B. Sooryanarayana And B. Shanmukha. A note on metric dimension. Far East J. Appl. Math., 5(3):331-339, 2001.

[41] Ionn Tomescu. Discrepancies between metric dimension and partition dimension of a connected graph. Discrete Math., 308(22):5026-5031, 2008. http://dx.doi. org $/ 10.1016 / j . d i s c .2007 .08 .089$

[42] S. V. Yushmanov. Estimates for the metric dimension of a graph in terms of the diameters and the number of vertices. Vestnik Moskov. Univ. Ser. I Mat. Mekh., 103:68-70, 1987. 